\title{
MORE ON INDUCED MAPS ON $n$-FOLD SYMMETRIC PRODUCT SUSPENSIONS
}

\author{
Franco Barragán, Sergio Macías and Jesús F. Tenorio
}

Universidad Tecnológica de la Mixteca and Universidad Nacional Autónoma de México, México

Abstract. We continue the work initiated by the first named author in Induced maps on $n$-fold symmetric product suspensions, Topology Appl. 158 (2011), 1192-1205. We consider classes of maps not included in the mentioned paper, namely: almost monotone, atriodic, freely decomposable, joining, monotonically refinable, refinable, semi-confluent, semi-open, simple and strongly freely decomposable maps.

\section{Introduction}

We continue the work initiated by the first named author in [2]. We consider classes of maps not included in the mentioned paper, namely: Almost monotone, atriodic, freely decomposable, joining, monotonically refinable, refinable, semi-confluent, semi-open, simple and strongly freely decomposable maps.

Let $\mathcal{M}$ be one of the classes of maps between continua mentioned in the previous paragraph. We study the relationships between the following statements:
(1) $f \in \mathcal{M}$,
(2) $\mathcal{F}_{n}(f) \in \mathcal{M}$
(3) $\mathcal{S F}_{n}(f) \in \mathcal{M}$

The paper contains eleven sections. After the first two sections, each section deals with one of the classes of maps mentioned above, except: Section 6,

2010 Mathematics Subject Classification. 54B20, 54E40, 54F15.

Key words and phrases. Almost monotone map, atriodic map, continuum, $\varepsilon$-map, freely decomposable map, hyperspace, joining map, monotone map, monotonically refinable map, refinable map, semi-confluent map, semi-open map, simple map and strongly freely decomposable map. 
in which we work with both freely decomposable and strongly freely decomposable maps; and Section 8, in which we work with both, refinable and monotonically refinable maps.

\section{Definitions and notations}

If $Z$ is a metric space, then given a subset $A$ of $Z$, the interior of $A$ is denoted by $\operatorname{Int}_{Z}(A)$. If $\varepsilon>0$, then $\mathcal{V}_{\varepsilon}(A)$ denotes the set of points of $X$ whose distance to $A$ is less than $\varepsilon$. Also, $\operatorname{diam}(A)$ denotes the diameter of $A$.

Given a function $f: X \rightarrow Y$ between metric spaces, if $A$ is a subset of $X$, then $\left.f\right|_{A}$ denotes the restriction of $f$ to $A$.

A continuum is a nonempty compact, connected and metric space. A map is a continuous function.

Let $f: X \rightarrow Y$ be a surjective map between continua. The function $f$ is:

- almost monotone provided that for each subcontinuum with nonempty interior $Q$ of $Y, f^{-1}(Q)$ is connected.

- atriodic if for every subcontinuum $Q$ of $Y$, there exist two components $C$ and $D$ of $f^{-1}(Q)$ such that $f(C) \cup f(D)=Q$ and for each component $E$ of $f^{-1}(Q)$, we have that either $f(E)=Q$, or $f(E) \subset f(C)$ or $f(E) \subset f(D)$.

- an $\varepsilon$-map provided that $\varepsilon>0$ and $\operatorname{diam}\left(f^{-1}(y)\right)<\varepsilon$ for each $y \in Y$.

- freely decomposable if whenever $A$ and $B$ are proper subcontinua of $Y$ such that $Y=A \cup B$, then there exist two proper subcontinua $A^{\prime}$ and $B^{\prime}$ of $X$, such that $X=A^{\prime} \cup B^{\prime}, f\left(A^{\prime}\right) \subset A$ and $f\left(B^{\prime}\right) \subset B$.

- joining provided that for each subcontinuum $Q$ of $Y$ and for each two components $C$ and $D$ of $f^{-1}(Q)$, we have that $f(C) \cap f(D) \neq \emptyset$.

- monotone provided that $f^{-1}(Q)$ is connected, for each subcontinuum $Q$ of $Y$.

- monotonically refinable provided that for each $\varepsilon>0$, there exists a monotone $\varepsilon$-map $g: X \rightarrow Y$ such that $d(f(x), g(x))<\varepsilon$ for all $x \in X$.

- refinable if for every $\varepsilon>0$, there exists an $\varepsilon$-map $g: X \rightarrow Y$ such that $d(f(x), g(x))<\varepsilon$ for all $x \in X$.

- semi-confluent provided that for each subcontinuum $Q$ of $Y$ and for each two components $C$ and $D$ of $f^{-1}(Q)$, either $f(C) \subset f(D)$ or $f(D) \subset f(C)$.

- semi-open if for each open subset $U$ of $X, \operatorname{Int}_{Y}(f(U)) \neq \emptyset$.

- simple provided that $f^{-1}(y)$ has at most two points for all $y \in Y$.

- strongly freely decomposable if whenever $A$ and $B$ are proper subcontinua of $Y$ such that $Y=A \cup B$, we obtain that $f^{-1}(A)$ and $f^{-1}(B)$ are connected ${ }^{1}$.

${ }^{1}$ Strongly freely decomposable maps are also known in the literature as feebly monotone maps. 
Given a continuum $X$, we define its hyperspaces as the following sets:

$$
\begin{gathered}
2^{X}=\{A \subset X \mid A \text { is closed and nonempty }\} \\
\mathcal{C}_{n}(X)=\left\{A \in 2^{X} \mid A \text { has at most } n \text { components }\right\}, n \in \mathbb{N} ; \\
\mathcal{F}_{n}(X)=\left\{A \in 2^{X} \mid A \text { has at most } n \text { points }\right\}, n \in \mathbb{N} .
\end{gathered}
$$

We topologize these sets with the Hausdorff metric, $\mathcal{H}([15,(0.1)]) . \mathcal{C}_{n}(X)$ is the $n$-fold hyperspace of $X$ and $\mathcal{F}_{n}(X)$ is the $n$-fold symmetric product of $X$.

Let $A_{1}, \ldots, A_{m}$ be nonempty subsets of $X$. We define the set

$$
\left\langle A_{1}, \ldots, A_{m}\right\rangle=\left\{D \in 2^{X} \mid D \subset \cup_{i=1}^{m} A_{i} \text { and } D \cap A_{i} \neq \emptyset \text { for each } i\right\} \text {. }
$$

Note that this set is nonempty (for each $j \in\{1, \ldots, m\}$, let $a_{j} \in A_{j}$, then we have that $\left.\left\{a_{1}, \ldots, a_{m}\right\} \in\left\langle A_{1}, \ldots, A_{m}\right\rangle\right)$. It is known that the family $\left\{\left\langle U_{1}, \ldots, U_{l}\right\rangle \mid U_{1}, \ldots, U_{l}\right.$ are open subsets of $\left.X\right\}$ forms a base for a topology on $2^{X}$ called the Vietoris topology $([15,(0.11)])$. It is well known that the Vietoris topology and the topology induced by the Hausdorff metric coincide $([15,(0.13)])$.

Notation 2.1. Let $X$ be a continuum, let $U_{1}, \ldots, U_{m}$ be a finite family of open subsets of $X$ and let $n$ be a positive integer. Then $\left\langle U_{1}, \ldots, U_{m}\right\rangle_{n}$ denotes the set $\left\langle U_{1}, \ldots, U_{m}\right\rangle \cap \mathcal{F}_{n}(X)$.

Let $n$ be an integer greater than or equal to 2 . Then the $n$-fold symmetric product suspension $([1])$ of a continuum $X$, denoted by $\mathcal{S F}_{n}(X)$, is the quotient space $\mathcal{F}_{n}(X) / \mathcal{F}_{1}(X)$, with the quotient topology. The quotient map is denoted by $q_{X}^{n}: \mathcal{F}_{n}(X) \rightarrow \mathcal{S} \mathcal{F}_{n}(X)$ and we denote $q_{X}^{n}\left(\mathcal{F}_{1}(X)\right)$ by $F_{X}^{n}$.

Let $n$ be an integer greater than or equal to 2 , and let $X$ and $Y$ be continua. If $f: X \rightarrow Y$ is a map, then we define the function $\mathcal{F}_{n}(f): \mathcal{F}_{n}(X) \rightarrow$ $\mathcal{F}_{n}(Y)$ by $\mathcal{F}_{n}(f)(A)=f(A)$ for all $A \in \mathcal{F}_{n}(X)$, and it is called the induced map of $f$ on the $n$-fold symmetric products of $X$ and $Y$. Note that $\mathcal{F}_{n}(f)$ is continuous $([13,1.8 .23])$. We also define the function $\mathcal{S F}_{n}(f): \mathcal{S F}_{n}(X) \rightarrow$ $\mathcal{S F}_{n}(Y)([2])$ by

$$
\mathcal{S F}_{n}(f)(\chi)= \begin{cases}q_{Y}^{n}\left(\mathcal{F}_{n}(f)\left(\left(q_{X}^{n}\right)^{-1}(\chi)\right)\right), & \text { if } \chi \neq F_{X}^{n} \\ F_{Y}^{n}, & \text { if } \chi=F_{X}^{n}\end{cases}
$$

Note that, by $[6,4.3$, p. 126$], \mathcal{S F}_{n}(f)$ is continuous. In addition, the following diagram:

is commutative.

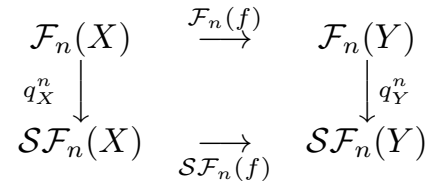




\section{Preliminary Results}

In this section we present the results needed for the rest of the paper. In particular, we prove a couple of theorems that relate the classes of maps defined above and quotient spaces (Theorems 3.2 and 3.3).

Let $X$ be a continuum and let $K$ be a subcontinuum of $X$. We denote by $X / K$ the quotient space obtained by shrinking $K$ to a point. Then $X / K$ is a continuum $([13,1.7 .3])$. Let $X$ and $Y$ be continua, let $K$ be a subcontinuum of $X$, and let $f: X \rightarrow Y$ be a surjective map. Consider the quotient spaces $X / K$ and $Y / f(K)$. Let $q_{X}: X \rightarrow X / K$ and $q_{Y}: Y \rightarrow Y / f(K)$ be the quotient maps. We denote $q_{X}(K)$ and $q_{Y}(f(K))$ by $K_{X}$ and $K_{Y}$, respectively. Note that $f$ induces a function $f_{\star}: X / K \rightarrow Y / f(K)([6,7.7$, p. 17]) given by

$$
f_{\star}(\chi)= \begin{cases}q_{Y}\left(f\left(\left(q_{X}\right)^{-1}(\chi)\right)\right), & \text { if } \chi \neq K_{X} \\ K_{Y}, & \text { if } \chi=K_{X} .\end{cases}
$$

The continuity of $f_{\star}$ follows from $[6,4.3, \mathrm{p} .126]$. Observe that the following diagram:

is commutative.

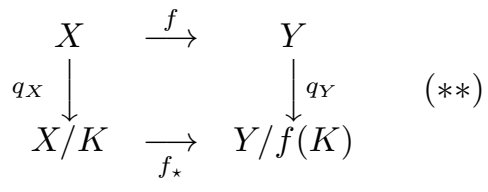

Proposition 3.1. Let $X$ and $Y$ be continua and let $K$ be a subcontinuum of $X$. If $f: X \rightarrow Y$ is a surjective map, the following hold:

(a) the maps $q_{X}$ and $q_{Y}$ are monotone;

(b) the maps $\left.q_{X}\right|_{X \backslash K}: X \backslash K \rightarrow X / K \backslash\left\{K_{X}\right\}$ and $\left.q_{Y}\right|_{Y \backslash f(K)}: Y \backslash f(K) \rightarrow$ $Y / f(K) \backslash\left\{K_{Y}\right\}$ are homeomorphisms;

(c) if $K$ and $f(K)$ are nowhere dense in $X$ and $Y$, respectively, then $q_{X}$ and $q_{Y}$ are semi-open maps;

(d) for each subcontinuum $\Gamma$ of $Y / f(K), f_{\star}^{-1}(\Gamma)=q_{X}\left(f^{-1}\left(q_{Y}^{-1}(\Gamma)\right)\right)$;

(e) if $\Delta$ and $\Gamma$ are proper subcontinua of $X / K$ such that $X / K=\Delta \cup \Gamma$, then $q_{X}^{-1}(\Delta)$ and $q_{X}^{-1}(\Gamma)$ are proper subcontinua of $X$ such that $X=$ $q_{X}^{-1}(\Delta) \cup q_{X}^{-1}(\Gamma)$;

(f) if $\Gamma$ is a subcontinuum of $X / K$ such that $\operatorname{Int}_{X / K}(\Gamma) \neq \emptyset$, then $q_{X}^{-1}(\Gamma)$ is a subcontinuum of $X$ such that $\operatorname{Int}_{X}\left(q_{X}^{-1}(\Gamma)\right) \neq \emptyset$;

$(g)$ if $G$ is a subcontinuum of $Y$ such that $G \cap f(K)=\emptyset$ and $D$ is a component of $f^{-1}(G)$, then $q_{X}(D)$ is a component of $f_{\star}^{-1}\left(q_{Y}(G)\right)$;

(h) if $G$ is a subcontinuum of $Y$ such that $G \cap f(K)=\emptyset$ and $\Delta$ is a component of $f_{\star}^{-1}\left(q_{Y}(G)\right)$, then $q_{X}^{-1}(\Delta)$ is a component of $f^{-1}(G)$;

(i) if $\Gamma$ is a subcontinuum of $Y / f(K)$ and $D$ is a component of $f^{-1}\left(q_{Y}^{-1}(\Gamma)\right)$, then $q_{X}(D)$ is a component of $f_{\star}^{-1}(\Gamma)$; 
(j) if $\Gamma$ is a subcontinuum of $Y / f(K)$ and $\Delta$ is a component of $f_{\star}{ }^{-1}(\Gamma)$, then there exists a component $D$ of $f^{-1}\left(q_{Y}^{-1}(\Gamma)\right)$ such that $q_{X}(D)=\Delta$.

Proof. Note that $(a)$ and $(b)$ follow from the definitions. Also, the proofs of $(c),(d),(e)$ and $(f)$ are simple.

To see $(g)$, let $G$ be a subcontinuum of $Y$ such that $G \cap f(K)=\emptyset$, and let $D$ be a component of $f^{-1}(G)$. Since $D \subset f^{-1}(G), q_{Y}(f(D)) \subset$ $q_{Y}(G)$. Then, since $(* *)$ is commutative, we have that $f_{\star}\left(q_{X}(D)\right) \subset q_{Y}(G)$. Let $\Delta$ be the component of $f_{\star}{ }^{-1}\left(q_{Y}(G)\right)$ containing $q_{X}(D)$. Hence, $D \subset$ $q_{X}^{-1}(\Delta) \subset q_{X}^{-1}\left(f_{\star}^{-1}\left(q_{Y}(G)\right)\right)$. Thus, since $(* *)$ is commutative, $D \subset q_{X}^{-1}(\Delta) \subset$ $f^{-1}\left(q_{Y}^{-1}\left(q_{Y}(G)\right)\right)$. Since $G \cap f(K)=\emptyset$ and $\left.q_{Y}\right|_{Y \backslash f(K)}$ is a homeomorphism (part $(b)$ ), we have that $D \subset q_{X}^{-1}(\Delta) \subset f^{-1}(G)$. Since $q_{X}$ is monotone (part $(a))$ and $D$ is a component of $f^{-1}(G)$, we obtain that $D=q_{X}^{-1}(\Delta)$. Hence, $q_{X}(D)=\Delta$. Therefore, $q_{X}(D)$ is a component of $f_{\star}{ }^{-1}\left(q_{Y}(G)\right)$.

To show $(h)$, let $G$ be a subcontinuum of $Y$ such that $G \cap f(K)=\emptyset$ and let $\Delta$ be a component of $f_{\star}{ }^{-1}\left(q_{Y}(G)\right)$. Since $\Delta \subset f_{\star}{ }^{-1}\left(q_{Y}(G)\right)$, $q_{X}^{-1}(\Delta) \subset q_{X}^{-1}\left(f_{\star}^{-1}\left(q_{Y}(G)\right)\right)$. Then, since $(* *)$ is commutative, $q_{X}^{-1}(\Delta) \subset$ $f^{-1}\left(q_{Y}^{-1}\left(q_{Y}(G)\right)\right)$. Since $G \cap f(K)=\emptyset$ and $\left.q_{Y}\right|_{Y \backslash f(K)}$ is a homeomorphism (part $(b)), q_{X}^{-1}(\Delta) \subset f^{-1}(G)$. Since $q_{X}$ is monotone (part $(a)$ ), $q_{X}^{-1}(\Delta)$ is connected. Let $D$ be the component of $f^{-1}(G)$ containing $q_{X}^{-1}(\Delta)$. Thus, $\Delta \subset q_{X}(D) \subset q_{X}\left(f^{-1}(G)\right)$. This implies that $f_{\star}\left(q_{X}(D)\right) \subset q_{Y}(G)$. Hence, $q_{X}(D) \subset f_{\star}^{-1}\left(q_{Y}(G)\right)$. Since $\Delta \subset q_{X}(D)$, we obtain that $\Delta=q_{X}(D)$. As a consequence of all this, we have that $D \subset q_{X}^{-1}(\Delta) \subset D$, and $D=q_{X}^{-1}(\Delta)$. Therefore, $q_{X}^{-1}(\Delta)$ is a component of $f^{-1}(G)$.

To prove $(i)$, let $\Gamma$ be a subcontinuum of $Y / f(K)$ and let $D$ be a component of $f^{-1}\left(q_{Y}^{-1}(\Gamma)\right)$. Since $D \subset f^{-1}\left(q_{Y}^{-1}(\Gamma)\right)$ and $(* *)$ commutes, $D \subset q_{X}^{-1}\left(f_{\star}^{-1}(\Gamma)\right)$. Thus, $q_{X}(D) \subset f_{\star}^{-1}(\Gamma)$. Let $\Delta$ be the component of $f_{\star}^{-1}(\Gamma)$ containing $q_{X}(D)$. Then, since $(* *)$ is commutative, we obtain that $D \subset q_{X}^{-1}(\Delta) \subset f^{-1}\left(q_{Y}^{-1}(\Gamma)\right)$. Hence, since $q_{X}^{-1}(\Delta)$ is connected ( $q_{X}$ is monotone $($ part $(a)))$, we have that $D=q_{X}^{-1}(\Delta)$. This implies that $q_{X}(D)=\Delta$. Therefore, $q_{X}(D)$ is a component of $f_{\star}^{-1}(\Gamma)$.

To see $(j)$, let $\Gamma$ be a subcontinuum of $Y / f(K)$ and let $\Delta$ be a component of $f_{\star}{ }^{-1}(\Gamma)$. Since $\Delta \subset f_{\star}{ }^{-1}(\Gamma)$ and $(* *)$ is commutative, we obtain that $q_{X}^{-1}(\Delta) \subset f^{-1}\left(q_{Y}^{-1}(\Gamma)\right)$. Note that $q_{X}^{-1}(\Delta)$ is connected $\left(q_{X}\right.$ is monotone (part $(a))$ ). Let $D$ be the component of $f^{-1}\left(q_{Y}^{-1}(\Gamma)\right)$ containing $q_{X}^{-1}(\Delta)$. Hence, by part $(d), \Delta \subset q_{X}(D) \subset q_{X}\left(f^{-1}\left(q_{Y}^{-1}(\Gamma)\right)\right)=f_{\star}^{-1}(\Gamma)$. Since $\Delta$ is a component of $f_{\star}{ }^{-1}(\Gamma)$, we obtain that $\Delta=q_{X}(D)$.

Theorem 3.2. Let $X$ and $Y$ be continua, let $K$ be a subcontinuum of $X$ and let $f: X \rightarrow Y$ be a surjective map. Let $\mathcal{M}$ be one of the following classes of maps: almost monotone, atriodic, freely decomposable, joining, semi-confluent and strongly freely decomposable. If $f \in \mathcal{M}$, then $f_{\star} \in \mathcal{M}$. 
Proof. It is proved in [5, Proposition 7.5] that if $f$ is either almost monotone or strongly freely decomposable, then $f_{\star}$ is almost monotone or strongly freely decomposable, respectively.

Suppose $f$ is atriodic. Let $\Gamma$ be a subcontinuum of $Y / f(K)$. Since $q_{Y}$ is monotone (part (a) of Proposition 3.1), $q_{Y}^{-1}(\Gamma)$ is a subcontinuum of $Y$. Since $f$ is atriodic, there exist two components $D$ and $L$ of $f^{-1}\left(q_{Y}^{-1}(\Gamma)\right)$ such that $f(D) \cup f(L)=q_{Y}^{-1}(\Gamma)$ and for each component $S$ of $f^{-1}\left(q_{Y}^{-1}(\Gamma)\right)$, we have that either $f(S)=q_{Y}^{-1}(\Gamma)$ or $f(S) \subset f(D)$ or $f(S) \subset f(L)$. By part $(i)$ of Proposition 3.1, $\Delta=q_{X}(D)$ and $\Lambda=q_{X}(L)$ are components of $f_{\star}^{-1}(\Gamma)$. Since $f(D) \cup$ $f(L)=q_{Y}^{-1}(\Gamma)$, we obtain that $q_{Y}(f(D)) \cup q_{Y}(f(L))=q_{Y}\left(q_{Y}^{-1}(\Gamma)\right)$. Hence, since $(* *)$ is commutative and $q_{Y}$ is surjective, $f_{\star}\left(q_{X}(D)\right) \cup f_{\star}\left(q_{X}(L)\right)=\Gamma$. This implies that $f_{\star}(\Delta) \cup f_{\star}(\Lambda)=\Gamma$. Now, let $\Sigma$ be a component of $f_{\star}^{-1}(\Gamma)$. By part $(j)$ of Proposition 3.1, there exists a component $S$ of $f^{-1}\left(q_{Y}^{-1}(\Gamma)\right)$ such that $q_{X}(S)=\Sigma$. Since $f$ is atriodic, either $f(S)=q_{Y}^{-1}(\Gamma)$ or $f(S) \subset f(D)$ or $f(S) \subset f(L)$. Then either $q_{Y}(f(S))=q_{Y}\left(q_{Y}^{-1}(\Gamma)\right)$ or $q_{Y}(f(S)) \subset q_{Y}(f(D))$ or $q_{Y}(f(S)) \subset q_{Y}(f(L))$. Thus, since $(* *)$ is commutative and $q_{Y}$ is surjective, we have that either $f_{\star}\left(q_{X}(S)\right)=\Gamma$ or $f_{\star}\left(q_{X}(S)\right) \subset f_{\star}\left(q_{X}(D)\right)$ or $f_{\star}\left(q_{X}(S)\right) \subset f_{\star}\left(q_{X}(L)\right)$. This implies that either $f_{\star}(\Sigma)=\Gamma$ or $f_{\star}(\Sigma) \subset f_{\star}(\Delta)$ or $f_{\star}(\Sigma) \subset f_{\star}(\Lambda)$. Therefore, $f_{\star}$ is atriodic.

Assume $f$ is freely decomposable. Let $\Delta$ and $\Gamma$ be proper subcontinua of $Y / f(K)$ such that $Y / f(K)=\Delta \cup \Gamma$. By part $(e)$ of Proposition 3.1, $q_{Y}^{-1}(\Delta)$ and $q_{Y}^{-1}(\Gamma)$ are proper subcontinua of $Y$ such that $Y=q_{Y}^{-1}(\Delta) \cup q_{Y}^{-1}(\Gamma)$. Since $f$ is freely decomposable, there exist two proper subcontinua $D$ and $G$ of $X$ such that $X=D \cup G$ and $f(D) \subset q_{Y}^{-1}(\Delta)$ and $f(G) \subset q_{Y}^{-1}(\Gamma)$. Let $\Delta^{\prime}=q_{X}(D)$ and let $\Gamma^{\prime}=q_{X}(G)$. Then $\Delta^{\prime}$ and $\Gamma^{\prime}$ are proper subcontinua of $X / K$ such that $X / K=\Delta^{\prime} \cup \Gamma^{\prime}$. Since $(* *)$ is commutative, we obtain that $f_{\star}\left(\Delta^{\prime}\right) \subset \Delta$ and $f_{\star}\left(\Gamma^{\prime}\right) \subset \Gamma$. Therefore, $f_{\star}$ is freely decomposable.

Suppose $f$ is joining. Let $\Gamma$ be a subcontinuum of $Y / f(K)$ and let $\Delta$ and $\Lambda$ be two components of $f_{\star}^{-1}(\Gamma)$. By part $(j)$ of Proposition 3.1, there exist two components $D$ and $L$ of $f^{-1}\left(q_{Y}^{-1}(\Gamma)\right)$ such that $\Delta=q_{X}(D)$ and $\Lambda=q_{X}(L)$. Since $f$ is joining, $f(D) \cap f(L) \neq \emptyset$. Since $q_{Y}(f(D) \cap f(L)) \subset$ $q_{Y}(f(D)) \cap q_{Y}(f(L))$, we have that $q_{Y}(f(D)) \cap q_{Y}(f(L)) \neq \emptyset$. Then, since $(* *)$ is commutative, $f_{\star}\left(q_{X}(D)\right) \cap f_{\star}\left(q_{X}(L)\right) \neq \emptyset$; i.e. $f_{\star}(\Delta) \cap f_{\star}(\Gamma) \neq \emptyset$. Therefore, $f_{\star}$ is joining.

Assume $f$ is semi-confluent. Let $\Gamma$ be a subcontinuum of $Y / f(K)$ and let $\Delta$ and $\Lambda$ be two components of $f_{\star}^{-1}(\Gamma)$. By part $(j)$ of Proposition 3.1, there exist two components $D$ and $L$ of $f^{-1}\left(q_{Y}^{-1}(\Gamma)\right)$ such that $\Delta=q_{X}(D)$ and $\Lambda=q_{X}(L)$. Since $f$ is semi-confluent, either $f(D) \subset f(L)$ or $f(L) \subset f(D)$. Then either $q_{Y}(f(D)) \subset q_{Y}(f(L))$ or $q_{Y}(f(L)) \subset q_{Y}(f(D))$. Hence, since $(* *)$ is commutative, either $f_{\star}\left(q_{X}(D)\right) \subset f_{\star}\left(q_{X}(L)\right)$ or $f_{\star}\left(q_{X}(L)\right) \subset f_{\star}\left(q_{X}(D)\right)$; i.e. either $f_{\star}(\Delta) \subset f_{\star}(\Lambda)$ or $f_{\star}(\Lambda) \subset f_{\star}(\Delta)$. Therefore, $f_{\star}$ is semi-confluent. 
Theorem 3.3. Let $X$ and $Y$ be continua and let $f: X \rightarrow Y$ be a surjective map. Suppose $K$ is a subcontinuum of $X$ such that $K$ and $f(K)$ are nowhere dense in $X$ and $Y$, respectively. Then $f$ is semi-open if and only if $f_{\star}$ is semi-open.

Proof. Suppose $f$ is semi-open. Let $\mathcal{U}$ be a nonempty open subset of $X / K$. Since $q_{X}$ is continuous, $q_{X}^{-1}(\mathcal{U})$ is a nonempty open subset of $X$. Since $f$ is semi-open, $\operatorname{Int}_{Y}\left(f\left(q_{X}^{-1}(\mathcal{U})\right)\right) \neq \emptyset$. Since $f(K)$ is nowhere dense in $Y$, by part $(c)$ of Proposition 3.1, $q_{Y}$ is semi-open. Then

$$
\operatorname{Int}_{Y / f(K)}\left(q_{Y}\left(\operatorname{Int}_{Y}\left(f\left(q_{X}^{-1}(\mathcal{U})\right)\right)\right)\right) \neq \emptyset .
$$

Since $(* *)$ is commutative, we have that $\operatorname{Int}_{Y / f(K)}\left(q_{Y}\left(\operatorname{Int}_{Y}\left(f\left(q_{X}^{-1}(\mathcal{U})\right)\right)\right)\right) \subset$ $\operatorname{Int}_{Y / f(K)}\left(f_{\star}(\mathcal{U})\right)$. Therefore, $f_{\star}$ is semi-open.

Assume that $f_{\star}$ is semi-open. Let $U$ be a nonempty open subset of $X$. Since $K$ is nowhere dense in $X$, there exists a nonempty open subset $V$ of $X$ such that $V \subset U \backslash K$. Hence, by part (b) of Proposition 3.1, $q_{X}(V)$ is a nonempty open subset of $X / K$. Since $f_{\star}$ is semi-open, $\operatorname{Int}_{Y / f(K)}\left(f_{\star}\left(q_{X}(V)\right)\right) \neq \emptyset$. Thus, there exists a nonempty open subset $\mathcal{W}$ of $Y / f(K)$ such that $\mathcal{W} \subset \operatorname{Int}_{Y / f(K)}\left(f_{\star}\left(q_{X}(V)\right)\right) \backslash\left\{K_{Y}\right\}$. Then, by part $(b)$ of Proposition 3.1 and the commutativity of $(* *), q_{Y}^{-1}(\mathcal{W}) \subset f(U)$. Hence, $\operatorname{Int}_{Y}(f(U)) \neq \emptyset$. Therefore, $f$ is semi-open.

The following result is [2, Theorem 3.3].

Lemma 3.4. Let $X$ and $Y$ be continua and let $n$ be an integer greater than or equal to 2 . If $f: X \rightarrow Y$ is a map, then the following are equivalent:

(1) $f: X \rightarrow Y$ is surjective;

(2) $\mathcal{F}_{n}(f): \mathcal{F}_{n}(X) \rightarrow \mathcal{F}_{n}(Y)$ is surjective;

(3) $\mathcal{S F}_{n}(f): \mathcal{S F}_{n}(X) \rightarrow \mathcal{S F}_{n}(Y)$ is surjective.

REMARK 3.5. As a consequence of Lemma 3.4 we obtain that all the maps we are working with are surjective.

Lemma 3.6. Let $X$ and $Y$ be continua, let $n$ be an integer greater than or equal to 2 , and let $f: X \rightarrow Y$ be a surjective map. If $B_{1}, \ldots, B_{n}$ are pairwise disjoint subcontinua of $Y$ and $E_{j}$ is a component of $f^{-1}\left(B_{j}\right)$, for each $j \in$ $\{1, \ldots, n\}$, then $\left\langle E_{1}, \ldots, E_{n}\right\rangle_{n}$ is a component of $\mathcal{F}_{n}(f)^{-1}\left(\left\langle B_{1}, \ldots, B_{n}\right\rangle_{n}\right)$.

Proof. Let $B_{1}, \ldots, B_{n}$ be pairwise disjoint subcontinua of $Y$ and let $E_{j}$ be a component of $f^{-1}\left(B_{j}\right)$, for each $j \in\{1, \ldots, n\}$. Then $\left\langle E_{1}, \ldots, E_{n}\right\rangle_{n}$ and $\left\langle B_{1}, \ldots, B_{n}\right\rangle_{n}$ are subcontinua of $\mathcal{F}_{n}(X)$ and $\mathcal{F}_{n}(Y)$, respectively ([14, Lemma 1]). Clearly, $\mathcal{F}_{n}(f)\left(\left\langle E_{1}, \ldots, E_{n}\right\rangle_{n}\right) \subset\left\langle B_{1}, \ldots, B_{n}\right\rangle_{n}$. Let $\mathcal{E}$ be the component of $\mathcal{F}_{n}(f)^{-1}\left(\left\langle B_{1}, \ldots, B_{n}\right\rangle_{n}\right)$ containing $\left\langle E_{1}, \ldots, E_{n}\right\rangle_{n}$. Then $\bigcup \mathcal{E}$ has at most $n$ components $([13,6.1 .2])$. Since $\bigcup_{j=1}^{n} E_{j} \subset \bigcup \mathcal{E}$ and $E_{j} \cap E_{k}=\emptyset$ if $j \neq k$, we obtain that $\bigcup \mathcal{E}$ has exactly $n$ components, say $E_{1}^{\prime}, \ldots, E_{n}^{\prime}$. Without loss of generality, we assume that $E_{j} \subset E_{j}^{\prime}, j \in\{1, \ldots, n\}$. Clearly, 
$f(\bigcup \mathcal{E}) \subset \bigcup_{j=1}^{n} B_{j}$. Since $E_{j}$ is a component of $f^{-1}\left(B_{j}\right)$, we have that $\bigcup \mathcal{E}=$ $\bigcup_{j=1}^{n} E_{j}$, and $E_{j}^{\prime}=E_{j}$ for all $j \in\{1, \ldots, n\}$.

Let $A \in \mathcal{E}$. Then $A \cap E_{j} \neq \emptyset$ for every $j \in\{1, \ldots, n\}$ ([8, Lemma 3.1]) and $A \subset \bigcup_{j=1}^{n} E_{j}$. This implies that $A \in\left\langle E_{1}, \ldots, E_{n}\right\rangle_{n}$. Hence, $\left\langle E_{1}, \ldots, E_{n}\right\rangle_{n}=$ $\mathcal{E}$. Therefore, $\left\langle E_{1}, \ldots, E_{n}\right\rangle_{n}$ is a component of $\mathcal{F}_{n}(f)^{-1}\left(\left\langle B_{1}, \ldots, B_{n}\right\rangle_{n}\right)$.

Lemma 3.7. Let $X$ and $Y$ be continua, let $n$ be an integer greater than or equal to 2 , and let $f: X \rightarrow Y$ be a surjective map. If $\mathcal{S F}_{n}(f)^{-1}\left(F_{Y}^{n}\right)=\left\{F_{X}^{n}\right\}$, then $f$ is a homeomorphism.

Proof. If $f$ is not one-to-one, then there exist two distinct points $x_{1}$ and $x_{2}$ in $X$ such that $f\left(x_{1}\right)=f\left(x_{2}\right)$. Then $q_{X}^{n}\left(\left\{x_{1}, x_{2}\right\}\right)$ belongs to $\mathcal{S F}_{n}(f)^{-1}\left(F_{Y}^{n}\right)$ and $\mathcal{S F}_{n}(f)^{-1}\left(F_{Y}^{n}\right) \neq\left\{F_{X}^{n}\right\}$.

\section{Almost monotone maps}

THEOREM 4.1. Let $X$ and $Y$ be continua, let $n$ be an integer greater than or equal to 2, and let $f: X \rightarrow Y$ be a surjective map. Consider the following statements:

(1) $f$ is almost monotone;

(2) $\mathcal{F}_{n}(f)$ is almost monotone;

(3) $\mathcal{S F}_{n}(f)$ is almost monotone.

Then (2) implies (1) and (3).

Proof. Suppose that $\mathcal{F}_{n}(f)$ is almost monotone, we see that $f$ is almost monotone. To this end, let $B$ be a subcontinuum of $Y$ with nonempty interior. Then $\langle B\rangle_{n}$ is connected ([14, Lemma 1]) and it is easy to see that it is also closed. Hence, $\langle B\rangle_{n}$ is a subcontinuum of $\mathcal{F}_{n}(Y)$. Since $\left\langle\operatorname{Int}_{Y}(B)\right\rangle_{n} \subset\langle B\rangle_{n}$, we have that $\operatorname{Int}_{\mathcal{F}_{n}(Y)}\left(\langle B\rangle_{n}\right) \neq \emptyset$. Since $\mathcal{F}_{n}(f)$ is almost monotone, $\mathcal{F}_{n}(f)^{-1}\left(\langle B\rangle_{n}\right)$ is a connected subset of $\mathcal{F}_{n}(X)$. Hence, $\mathcal{F}_{n}(f)^{-1}\left(\langle B\rangle_{n}\right)$ is a subcontinuum of $\mathcal{F}_{n}(X)$. Thus, $\cup \mathcal{F}_{n}(f)^{-1}\left(\langle B\rangle_{n}\right)$ is a closed subset of $X$. Observe that $\cup \mathcal{F}_{n}(f)^{-1}\left(\langle B\rangle_{n}\right)=f^{-1}(B)$, and $\emptyset \neq \mathcal{F}_{n}(f)^{-1}\left(\langle B\rangle_{n}\right) \cap \mathcal{F}_{1}(X) \subset \mathcal{F}_{n}(f)^{-1}\left(\langle B\rangle_{n}\right) \cap \mathcal{C}_{1}(X)$. Thus, $f^{-1}(B)$ is connected $([15,(1.49)])$. Therefore, $f$ is almost monotone.

It follows from Theorem 3.2 that if $\mathcal{F}_{n}(f)$ is almost monotone, then $\mathcal{S F}_{n}(f)$ is almost monotone.

Remark 4.2. In Theorem 4.1, when the range space $Y$ is locally connected and $n$ is greater than or equal to 3 , we also have that (3) implies (1) and (2), for this see Corollary 6.3. In fact, we have the following:

TheOrem 4.3. Let $X$ and $Y$ be continua, where $Y$ is locally connected. Then a surjective map $f: X \rightarrow Y$ is almost monotone if and only if $f$ is monotone. 
Proof. Clearly, if $f$ is monotone, then $f$ is almost monotone. Suppose $f$ is almost monotone. Let $y$ be a point of $Y$. Since $Y$ is locally connected, there exists a sequence $\left\{K_{m}\right\}_{m=1}^{\infty}$ of locally connected subcontinua of $Y$ such that $\{y\}=\bigcap_{m=1}^{\infty} K_{m}$, and for all positive integer $m, y \in \operatorname{Int}_{Y}\left(K_{m}\right)$ and $K_{m+1} \subset K_{m}$. Since $f$ is almost montonote, $f^{-1}\left(K_{m}\right)$ is connected. Hence, $f^{-1}(y)=\bigcap_{m=1}^{\infty} f^{-1}\left(K_{m}\right)$ is connected $([13,1.7 .2])$. Therefore, $f$ is monotone $([13,2.1 .12])$.

Questions 4.4. Let $X$ and $Y$ be continua, let $n$ be an integer greater than or equal to 2 , and let $f: X \rightarrow Y$ be a surjective map.

(i) If $f$ is almost monotone, then is $\mathcal{F}_{n}(f)$ almost monotone?

(ii) If $f$ is almost monotone, then is $\mathcal{S F}_{n}(f)$ almost monotone?

(iii) If $\mathcal{S F}_{n}(f)$ is almost monotone and $\mathcal{S F}_{n}(Y)$ is not locally connected, then is $f$ almost monotone?

(iv) If $\mathcal{S F}_{n}(f)$ is almost monotone and $\mathcal{S F}_{n}(Y)$ is not locally connected, then is $\mathcal{F}_{n}(f)$ almost monotone?

REMARK 4.5. Note that by Theorem 4.3 and [2, Theorem 4.1], questions (i) and (ii) have positive answers when $Y$ is locally connected.

\section{Atriodic MAPS}

Theorem 5.1. Let $X$ and $Y$ be continua, let $n$ be an integer greater than or equal to 2, and let $f: X \rightarrow Y$ be a surjective map. Consider the following statements:

(1) $f$ is atriodic;

(2) $\mathcal{F}_{n}(f)$ is atriodic;

(3) $\mathcal{S F}_{n}(f)$ is atriodic.

Then (2) implies (1) and (3); also (3) implies (1).

Proof. Suppose $\mathcal{F}_{n}(f)$ is atriodic, we prove that $f$ is atriodic. Let $K$ be a subcontinuum of $Y$. If $K=Y$, then $f^{-1}(K)$ has only one component, namely $X$, and $f(X)=K$. Suppose $K \neq Y$ and let $y_{1}, \ldots, y_{n-1}$ be $n-1$ distinct points in $Y \backslash K$. Let

$$
\mathcal{K}=\left\{\left\{y_{1}, \ldots, y_{n-1}\right\} \cup\{k\} \mid k \in K\right\} .
$$

Observe that $\mathcal{K}$ is a subcontinuum of $\mathcal{F}_{n}(Y)$ and $\mathcal{K} \cap \mathcal{F}_{1}(Y)=\emptyset$. Since $\mathcal{F}_{n}(f)$ is atriodic, there exist two components $\mathcal{C}$ and $\mathcal{D}$ of $\mathcal{F}_{n}(f)^{-1}(\mathcal{K})$ such that $\mathcal{F}_{n}(f)(\mathcal{C}) \cup \mathcal{F}_{n}(f)(\mathcal{D})=\mathcal{K}$ and for each component $\mathcal{E}$ of $\mathcal{F}_{n}(f)^{-1}(\mathcal{K})$ either $\mathcal{F}_{n}(f)(\mathcal{E})=\mathcal{K}$ or $\mathcal{F}_{n}(f)(\mathcal{E}) \subset \mathcal{F}_{n}(f)(\mathcal{C})$ or $\mathcal{F}_{n}(f)(\mathcal{E}) \subset \mathcal{F}_{n}(f)(\mathcal{D})$. Note that $\cup \mathcal{C} \subset\left(\bigcup_{j=1}^{n-1} f^{-1}\left(y_{j}\right)\right) \cup f^{-1}(K), \cup \mathcal{C} \cap f^{-1}\left(y_{j}\right) \neq \emptyset(j \in\{1, \ldots, n-1\})$, and $\bigcup \mathcal{C} \cap f^{-1}(K) \neq \emptyset$. Since $\bigcup \mathcal{C}$ has at most $n$ components $([13,6.1 .2])$, we have that $\bigcup \mathcal{C}$ has exactly $n$ components, say $C_{1}, \ldots, C_{n}$. Without loss of generality, we assume that for each $j \in\{1, \ldots, n-1\}, C_{j} \subset f^{-1}\left(y_{j}\right)$ and 
$C_{n} \subset f^{-1}(K)$. Let $C$ be the component of $f^{-1}(K)$ containing $C_{n}$. Similarly, $\bigcup \mathcal{D}$ has exactly $n$ components, say $D_{1}, \ldots, D_{n}$. Without loss of generality, we assume that for each $j \in\{1, \ldots, n-1\}, D_{j} \subset f^{-1}\left(y_{j}\right)$ and $D_{n} \subset f^{-1}(K)$. Let $D$ be the component of $f^{-1}(K)$ containing $D_{n}$.

We show that $f(C) \cup f(D)=K$. Note that $f(C) \cup f(D) \subset K$. Let $k \in K$. Then $\left\{y_{1}, \ldots, y_{n-1}\right\} \cup\{k\} \in \mathcal{K}$. Assume that $\left\{y_{1}, \ldots, y_{n-1}\right\} \cup\{k\} \in$ $\mathcal{F}_{n}(f)(\mathcal{C})$. Hence, there exists an element $A \in \mathcal{C}$ such that $\mathcal{F}_{n}(f)(A)=$ $\left\{y_{1}, \ldots, y_{n-1}\right\} \cup\{k\}$. Thus, $f(A)=\left\{y_{1}, \ldots, y_{n-1}\right\} \cup\{k\}$. This implies that $k \in f(A) \subset f(\bigcup \mathcal{C})=\bigcup_{j=1}^{n} f\left(C_{j}\right)$. Since $k \notin\left\{y_{1}, \ldots, y_{n-1}\right\}, k \in f\left(C_{n}\right) \subset$ $f(C)$. Similarly, if $\left\{y_{1}, \ldots, y_{n-1}\right\} \cup\{k\} \in \mathcal{F}_{n}(f)(\mathcal{D})$, then $k \in f\left(C_{n}\right) \subset f(D)$. Hence, $K \subset f(C) \cup f(D)$, and $f(C) \cup f(D)=K$.

Let $E$ be a component of $f^{-1}(K)$ and, for each $j \in\{1, \ldots, n-1\}$, let $E_{j}$ be a component of $f^{-1}\left(y_{j}\right)$. Then $\mathcal{E}=\left\langle E_{1}, \ldots, E_{n-1}, E\right\rangle_{n}$ is a component of $\mathcal{F}_{n}(f)^{-1}(\mathcal{K})$, by Lemma 3.6. Since $\mathcal{F}_{n}(f)$ is atriodic, either $\mathcal{F}_{n}(f)(\mathcal{E})=\mathcal{K}$ or $\mathcal{F}_{n}(f)(\mathcal{E}) \subset \mathcal{F}_{n}(f)(\mathcal{C})$ or $\mathcal{F}_{n}(f)(\mathcal{E}) \subset \mathcal{F}_{n}(f)(\mathcal{D})$

Suppose $\mathcal{F}_{n}(f)(\mathcal{E})=\mathcal{K}$, we prove that $f(E)=K$. Clearly, $f(E) \subset K$. Let $k \in K$. Then $\left\{y_{1}, \ldots, y_{n-1}\right\} \cup\{k\} \in \mathcal{K}$. Hence, there exists $L \in \mathcal{E}$ such that $\mathcal{F}_{n}(f)(L)=\left\{y_{1}, \ldots, y_{n-1}\right\} \cup\{k\}$; i.e. $f(L)=\left\{y_{1}, \ldots, y_{n-1}\right\} \cup\{k\}$. Thus, $k \in f(L)$. Since $k \notin\left\{y_{1}, \ldots, y_{n-1}\right\}, k \in f(E)$. Therefore, $f(E)=K$.

Assume $\mathcal{F}_{n}(f)(\mathcal{E}) \subset \mathcal{F}_{n}(f)(\mathcal{C})$, we show that $f(E) \subset f(C)$. Let $e \in E$. For each $j \in\{1, \ldots, n-1\}$, let $e_{j} \in E_{j}$. Then $\left\{e_{1}, \ldots, e_{n-1}, e\right\} \in \mathcal{E}$. Hence, $\mathcal{F}_{n}(f)\left(\left\{e_{1}, \ldots, e_{n-1}, e\right\}\right) \in \mathcal{F}_{n}(f)(\mathcal{C})$. Then there exists $C^{\prime} \in \mathcal{C}$ such that $\mathcal{F}_{n}(f)\left(C^{\prime}\right)=\mathcal{F}_{n}(f)\left(\left\{e_{1}, \ldots, e_{n-1}, e\right\}\right)$; i.e. $f\left(C^{\prime}\right)=f\left(\left\{e_{1}, \ldots, e_{n-1}, e\right\}\right)$. In particular, $f(e) \in f\left(C^{\prime}\right)$. Since $C^{\prime} \subset \bigcup \mathcal{C}=\bigcup_{j=1}^{n} C_{j}$ and $e \notin\left\{e_{1}, \ldots, e_{n-1}\right\}$, we obtain that $f(e) \in f\left(C_{n}\right) \subset f(C)$. Therefore, $f(E) \subset f(C)$.

Similarly, if $\mathcal{F}_{n}(f)(\mathcal{E}) \subset \mathcal{F}_{n}(f)(\mathcal{D})$, then $f(E) \subset f(D)$. Therefore, $f$ is an atriodic map.

Suppose $\mathcal{S F}_{n}(f)$ is atriodic, we see that $f$ is atriodic. The proof is similar to the previous one, we include the details for the convenience of the reader. Let $K$ be a subcontinuum of $Y$. If $K=Y$, then $f^{-1}(K)$ has only one component, namely $X$, and $f(X)=K$. Suppose $K \neq Y$ and let $y_{1}, \ldots, y_{n-1}$ be $n-1$ distinct points in $Y \backslash K$. Let

$$
\mathcal{K}=\left\{\left\{y_{1}, \ldots, y_{n-1}\right\} \cup\{k\} \mid k \in K\right\} .
$$

Observe that $\mathcal{K}$ is a subcontinuum of $\mathcal{F}_{n}(Y)$ and $\mathcal{K} \cap \mathcal{F}_{1}(Y)=\emptyset$. Then $q_{Y}^{n}(\mathcal{K})$ is a subcontinuum of $\mathcal{S F}_{n}(Y) \backslash\left\{F_{Y}^{n}\right\}$. Since $\mathcal{S F}_{n}(f)$ is atriodic, there exist two components $\mathfrak{C}$ and $\mathfrak{D}$ of $\mathcal{S F}_{n}(f)^{-1}\left(q_{Y}^{n}(\mathcal{K})\right)$ such that $\mathcal{S F}_{n}(f)(\mathfrak{C}) \cup$ $\mathcal{S F}_{n}(f)(\mathfrak{D})=q_{Y}^{n}(\mathcal{K})$ and for each component $\mathfrak{E}$ of $\mathcal{S F}_{n}(f)^{-1}\left(q_{Y}^{n}(\mathcal{K})\right)$, we have that either $\mathcal{S F}_{n}(f)(\mathfrak{E})=q_{Y}^{n}(\mathcal{K})$ or $\mathcal{S F}_{n}(f)(\mathfrak{E}) \subset \mathcal{S} \mathcal{F}_{n}(f)(\mathfrak{C})$ or $\mathcal{S F}_{n}(f)(\mathfrak{E}) \subset$ $\mathcal{S} \mathcal{F}_{n}(f)(\mathfrak{D})$. By part $(h)$ of Proposition 3.1, we obtain that $\left(q_{X}^{n}\right)^{-1}(\mathfrak{C})$ and $\left(q_{X}^{n}\right)^{-1}(\mathfrak{D})$ are components of $\mathcal{F}_{n}(f)^{-1}(\mathcal{K})$. Also,

$$
\mathcal{F}_{n}(f)\left(\left(q_{X}^{n}\right)^{-1}(\mathfrak{C})\right) \cup \mathcal{F}_{n}(f)\left(\left(q_{X}^{n}\right)^{-1}(\mathfrak{D})\right)=\mathcal{K} .
$$


Note that $\bigcup\left(q_{X}^{n}\right)^{-1}(\mathfrak{C}) \subset\left(\bigcup_{j=1}^{n-1} f^{-1}\left(y_{j}\right)\right) \cup f^{-1}(K),\left(\left(q_{X}^{n}\right)^{-1}(\mathfrak{C})\right) \cap f^{-1}\left(y_{j}\right) \neq$ $\emptyset(j \in\{1, \ldots, n-1\})$, and $\left(\left(q_{X}^{n}\right)^{-1}(\mathfrak{C})\right) \cap f^{-1}(K) \neq \emptyset$. Since $\left(q_{X}^{n}\right)^{-1}(\mathfrak{C})$ has at most $n$ components $[13,6.1 .2]$, we have that $\left(q_{X}^{n}\right)^{-1}(\mathfrak{C})$ has exactly $n$ components, say $C_{1}, \ldots, C_{n}$. Without loss of generality, we assume that for each $j \in\{1, \ldots, n-1\}, C_{j} \subset f^{-1}\left(y_{j}\right)$ and $C_{n} \subset f^{-1}(K)$. Let $C$ be the component of $f^{-1}(K)$ containing $C_{n}$. Similarly, $\left(q_{X}^{n}\right)^{-1}(\mathfrak{D})$ has exactly $n$ components, say $D_{1}, \ldots, D_{n}$. Without loss of generality, we assume that for each $j \in\{1, \ldots, n-1\}, D_{j} \subset f^{-1}\left(y_{j}\right)$ and $D_{n} \subset f^{-1}(K)$. Let $D$ be the component of $f^{-1}(K)$ containing $D_{n}$.

We show that $f(C) \cup f(D)=K$. Note that $f(C) \cup f(D) \subset K$. Let $k \in K$. Then $\left\{y_{1}, \ldots, y_{n-1}\right\} \cup\{k\} \in \mathcal{K}$. Assume that $\left\{y_{1}, \ldots, y_{n-1}\right\} \cup\{k\} \in$ $\mathcal{F}_{n}(f)\left(\left(q_{X}^{n}\right)^{-1}(\mathfrak{C})\right)$. Hence, there exists an element $A \in\left(q_{X}^{n}\right)^{-1}(\mathfrak{C})$ such that $\mathcal{F}_{n}(f)(A)=\left\{y_{1}, \ldots, y_{n-1}\right\} \cup\{k\}$. Thus, $f(A)=\left\{y_{1}, \ldots, y_{n-1}\right\} \cup\{k\}$. This implies that $k \in f(A) \subset \bigcup \mathcal{F}_{n}(f)\left(\left(q_{X}^{n}\right)^{-1}(\mathfrak{C})\right)=\bigcup_{j=1}^{n} f\left(C_{j}\right)$. Since $k \notin\left\{y_{1}, \ldots, y_{n-1}\right\}, k \in f\left(C_{n}\right) \subset f(C)$. Similarly, if $\left\{y_{1}, \ldots, y_{n-1}\right\} \cup\{k\} \in$ $\mathcal{F}_{n}(f)\left(\left(q_{X}^{n}\right)^{-1}(\mathfrak{D})\right)$, then $k \in f\left(D_{n}\right) \subset f(D)$. Hence, $K \subset f(C) \cup f(D)$, and $f(C) \cup f(D)=K$.

Let $E$ be a component of $f^{-1}(K)$ and, for each $j \in\{1, \ldots, n-1\}$, let $E_{j}$ be a component of $f^{-1}\left(y_{j}\right)$. Then $\mathcal{E}=\left\langle E_{1}, \ldots, E_{n-1}, E\right\rangle_{n}$ is a component of $\mathcal{F}_{n}(f)^{-1}(\mathcal{K})$, by Lemma 3.6. Thus, by part $(g)$ of Proposition 3.1, $\mathfrak{E}=q_{X}^{n}(\mathcal{E})$ is a component of $\mathcal{S F}_{n}(f)^{-1}\left(q_{Y}^{n}(\mathcal{K})\right)$. Since $\mathcal{S F}_{n}(f)$ is atriodic, we have that either $\mathcal{S F}_{n}(f)(\mathfrak{E})=q_{Y}^{n}(\mathcal{K})$ or $\mathcal{S F}_{n}(f)(\mathfrak{E}) \subset \mathcal{S F}_{n}(f)(\mathfrak{C})$ or $\mathcal{S F}_{n}(f)(\mathfrak{E}) \subset$ $\mathcal{S F}_{n}(f)(\mathfrak{D})$. Hence, since $(*)$ commutes, we obtain that either $\mathcal{F}_{n}(f)(\mathcal{E})=\mathcal{K}$ or $q_{Y}^{n}\left(\mathcal{F}_{n}(f)(\mathcal{E})\right) \subset \mathcal{S} \mathcal{F}_{n}(f)(\mathfrak{C})$ or $q_{Y}^{n}\left(\mathcal{F}_{n}(f)(\mathcal{E})\right) \subset \mathcal{S} \mathcal{F}_{n}(f)(\mathfrak{D})$.

Suppose that $\mathcal{F}_{n}(f)(\mathcal{E})=\mathcal{K}$. We show that $f(E)=K$. Clearly, $f(E) \subset$ $K$. Let $k \in K$. Then $\left\{y_{1}, \ldots, y_{n-1}\right\} \cup\{k\} \in \mathcal{K}$. Hence, there exists $L \in \mathcal{E}$ such that $\mathcal{F}_{n}(f)(L)=\left\{y_{1}, \ldots, y_{n-1}\right\} \cup\{k\}$; i.e. $f(L)=\left\{y_{1}, \ldots, y_{n-1}\right\} \cup\{k\}$. Thus, $k \in f(L)$ and $k \in f(E)$. Therefore, $f(E)=K$.

Assume that $q_{Y}^{n}\left(\mathcal{F}_{n}(f)(\mathcal{E})\right) \subset \mathcal{S F}_{n}(f)(\mathfrak{C})$. We see that $f(E) \subset f(C)$. Let $e \in E$. For each $j \in\{1, \ldots, n-1\}$, let $e_{j} \in E_{j}$. Then $\left\{e_{1}, \ldots, e_{n-1}, e\right\} \in$ $\mathcal{E}$. Hence, $q_{Y}^{n}\left(\mathcal{F}_{n}(f)\left(\left\{e_{1}, \ldots, e_{n-1}, e\right\}\right)\right) \in q_{Y}^{n}\left(\mathcal{F}_{n}(f)(\mathcal{E})\right)$. This implies that $q_{Y}^{n}\left(\mathcal{F}_{n}(f)\left(\left\{e_{1}, \ldots, e_{n-1}, e\right\}\right)\right) \in \mathcal{S F}_{n}(f)(\mathfrak{C})$. Thus, there exists $\mathcal{C} \in \mathfrak{C}$ such that $\mathcal{S F}_{n}(f)(\mathcal{C})=q_{Y}^{n}\left(\mathcal{F}_{n}(f)\left(\left\{e_{1}, \ldots, e_{n-1}, e\right\}\right)\right)$. Hence, there exists $C^{\prime} \in \mathcal{F}_{n}(X) \backslash \mathcal{F}_{1}(X)$ such that $q_{X}^{n}\left(C^{\prime}\right)=\mathcal{C}$. Since $(*)$ commutes, it follows that $q_{Y}^{n}\left(\mathcal{F}_{n}(f)\left(C^{\prime}\right)\right)=q_{Y}^{n}\left(\mathcal{F}_{n}(f)\left(\left\{e_{1}, \ldots, e_{n-1}, e\right\}\right)\right)$. Thus, by part $(b)$ of Proposition 3.1, we obtain that $\mathcal{F}_{n}(f)\left(C^{\prime}\right)=\mathcal{F}_{n}(f)\left(\left\{e_{1}, \ldots, e_{n-1}, e\right\}\right)$; i.e. $f\left(C^{\prime}\right)=f\left(\left\{e_{1}, \ldots, e_{n-1}, e\right\}\right)$. In particular, $f(e) \in f\left(C^{\prime}\right)$. Since $C^{\prime} \subset \bigcup \bigcup\left(q_{X}^{n}\right)^{-1}(\mathfrak{C})=\bigcup_{j=1}^{n} C_{j}$, we obtain that $f(e) \in f\left(C_{n}\right) \subset f(C)$. Therefore, $f(E) \subset f(C)$.

Similarly, if $q_{Y}^{n}\left(\mathcal{F}_{n}(f)(\mathcal{E})\right) \subset \mathcal{S F}_{n}(f)(\mathfrak{D})$, we have that $f(E) \subset f(D)$. Therefore, $f$ is an atriodic map. 
It follows from Theorem 3.2 that if $\mathcal{F}_{n}(f)$ is atriodic, then $\mathcal{S F}_{n}(f)$ is atriodic.

Questions 5.2. Let $X$ and $Y$ be continua, let $n$ be an integer greater than or equal to 2 , and let $f: X \rightarrow Y$ be a surjective map.

(i) If $f$ is atriodic, then is $\mathcal{F}_{n}(f)$ atriodic?

(ii) If $f$ is atriodic, then is $\mathcal{S F}_{n}(f)$ atriodic?

(iii) If $\mathcal{S F}_{n}(f)$ is atriodic, then is $\mathcal{F}_{n}(f)$ atriodic?

\section{Freely decomposable And Strongly fReely DeComposable maps}

THEOREM 6.1. Let $X$ be a continuum and let $Y$ be locally connected continuum. Let $n$ be an integer greater than or equal to 2 , and let $f: X \rightarrow Y$ be a surjective map. Then the following are equivalent:

(1) $\mathcal{F}_{n}(f)$ is freely decomposable;

(2) $\mathcal{F}_{n}(f)$ is monotone;

(3) $\mathcal{S F}_{n}(f)$ is freely decomposable;

(4) $\mathcal{S F}_{n}(f)$ is monotone;

(5) $f$ is monotone.

Proof. Suppose $\mathcal{F}_{n}(f)$ is freely decomposable. Since $Y$ is locally connected, $\mathcal{F}_{n}(Y)$ is locally connected $([11$, Lemma 2$])$. Also, by [11, Corollary 5], for each $B \in \mathcal{F}_{n}(Y), \mathcal{F}_{n}(Y) \backslash\{B\}$ is connected. Hence, by [7, Theorem 7], we have that $\mathcal{F}_{n}(f)$ is monotone. It is clear that if $\mathcal{F}_{n}(f)$ is monotone, then $\mathcal{F}_{n}(f)$ is freely decomposable. By [2, Theorem 4.1], we have that $f$ is monotone if and only if $\mathcal{F}_{n}(f)$ is monotone.

Assume $\mathcal{S F}_{n}(f)$ is freely decomposable. Since $Y$ is locally connected, $\mathcal{S F}_{n}(Y)$ is locally connected ([1, Theorem 5.2]). Also, by [3, Theorem 3.5], for each $\chi \in \mathcal{S F}_{n}(Y), \mathcal{S F}_{n}(Y) \backslash\{\chi\}$ is connected. Thus, by [7, Theorem 7], we have that $\mathcal{S F}_{n}(f)$ is monotone. It is clear that if $\mathcal{S F}_{n}(f)$ is monotone, then $\mathcal{S F}_{n}(f)$ is freely decomposable. By [2, Theorem 4.1], we have that $f$ is monotone if and only if $\mathcal{S F}_{n}(f)$ is monotone.

TheOREM 6.2. Let $X$ and $Y$ be continua, let $n$ be an integer greater than or equal to 3 , and let $f: X \rightarrow Y$ be a surjective map. Then

(1) $\mathcal{F}_{n}(f)$ is almost monotone if and only if $\mathcal{F}_{n}(f)$ is strongly freely decomposable;

(2) $\mathcal{S} \mathcal{F}_{n}(f)$ is almost monotone if and only if $\mathcal{S F}_{n}(f)$ is strongly freely decomposable.

Proof. Clearly, every almost monotone map is strongly freely decomposable. Suppose $\mathcal{F}_{n}(f)\left(\mathcal{S F}_{n}(f)\right)$ is strongly freely decomposable. By [10, Theorem 8] ([1, Theorem 4.1]) $\mathcal{F}_{n}(X)\left(\mathcal{S F}_{n}(X)\right)$ is unicoherent. Therefore, by $\left[4\right.$, Theorem 4.2], $\mathcal{F}_{n}(f)\left(\mathcal{S F}_{n}(f)\right)$ is almost monotone.

As a consequence of Theorems 4.1, 6.1 and 6.2, we obtain: 
Corollary 6.3. Let $X$ be a continuum and let $Y$ be locally connected continuum. Let $n$ be an integer greater than or equal to 3 , and let $f: X \rightarrow Y$ be a surjective map. Then the following are equivalent:

(1) $\mathcal{F}_{n}(f)$ is freely decomposable;

(2) $\mathcal{F}_{n}(f)$ is strongly freely decomposable;

(3) $\mathcal{F}_{n}(f)$ is almost monotone;

(4) $\mathcal{F}_{n}(f)$ is monotone;

(5) $\mathcal{S F}_{n}(f)$ is freely decomposable;

(6) $\mathcal{S F}_{n}(f)$ is strongly freely decomposable;

(7) $\mathcal{S F}_{n}(f)$ is almost monotone;

(8) $\mathcal{S F}_{n}(f)$ is monotone;

(9) $f$ is monotone.

The following example shows a strongly freely decomposable map $f$ defined between locally connected continua such that neither $\mathcal{F}_{n}(f)$ nor $\mathcal{S F}_{n}(f)$ is freely decomposable for any integer $n$ greater than or equal to 3 .

ExAmple 6.4. Let $\mathcal{S}^{1}=\left\{(x, y) \in \mathbb{R}^{2} \mid x^{2}+y^{2}=1\right\}$ and let $f: \mathcal{S}^{1} \rightarrow[-1,1]$ be given by $f((x, y))=x$. Then $f$ is strongly freely decomposable but it is neither monotone nor almost monotone. Hence, by Corollary 6.3, for an integer $n$ greater than or equal to $3, \mathcal{F}_{n}(f)$ and $\mathcal{S F}_{n}(f)$ are not freely decomposable.

Theorem 6.5. Let $X$ and $Y$ be continua, let $n$ be an integer greater than or equal to 2, and let $f: X \rightarrow Y$ be a surjective map. Consider the following statements:

(1) $f$ is freely decomposable;

(2) $\mathcal{F}_{n}(f)$ is freely decomposable;

(3) $\mathcal{S F}_{n}(f)$ is freely decomposable.

Then (2) implies (3). Also, if $Y$ is locally connected and $n$ is greater than or equal to 3, then (2) implies (1), (3) implies (2) and (3) implies (1). Moreover, if $n$ is greater than or equal to $3,(1)$ does not imply (2) and (1) does not imply (3).

Proof. It follows from Theorem 3.2 that (2) implies (3). If $Y$ is locally connected and $n$ is greater than or equal to 3 , by Corollary 6.3 , we have that (2) implies (1), (3) implies (2) and (3) implies (1). From Example 6.4, we obtain that if $n$ is greater than or equal to 3, (1) does not imply (2) and (1) does not imply (3).

THEOREM 6.6. Let $X$ and $Y$ be continua, let $n$ be an integer greater than or equal to 2, and let $f: X \rightarrow Y$ be a surjective map. Consider the following statements: 
(1) $f$ is strongly freely decomposable;

(2) $\mathcal{F}_{n}(f)$ is strongly freely decomposable;

(3) $\mathcal{S F}_{n}(f)$ is strongly freely decomposable.

Then (2) implies (3). Also, if $n$ is greater than or equal to 3, (2) implies (1), (1) does not imply (2) and (1) does not imply (3). Moreover, if $Y$ is locally connected and $n$ is greater than or equal to 3, then (3) implies (1) and (3) implies (2).

Proof. It follows from Theorem 3.2 that $(2)$ implies $(3)$. If $\mathcal{F}_{n}(f)$ is strongly freely decomposable and $n$ is greater than or equal to 3 , then, by Theorem $6.2, \mathcal{F}_{n}(f)$ is almost monotone. Hence, by Theorem $4.1, f$ is almost monotone. Thus, $f$ is strongly freely decomposable.

By Example 6.4, we have that, when $n$ is greater than or equal to 3, neither (1) implies (2) nor (1) implies (3).

It follows from Corollary 6.3 that if $Y$ is locally connected and $n$ is greater than or equal to 3 , then (3) implies (1) and (3) implies (2).

Questions 6.7. Let $X$ and $Y$ be continua, let $n$ be an integer greater than or equal to 2 , and let $f: X \rightarrow Y$ be a surjective map.

(i) If $f$ is freely decomposable, then is $\mathcal{F}_{2}(f)$ freely decomposable?

(ii) If $f$ is freely decomposable, then is $\mathcal{S F}_{2}(f)$ freely decomposable?

(iii) If $\mathcal{F}_{n}(f)$ is freely decomposable, then is $f$ freely decomposable?

(iv) If $\mathcal{S F}_{n}(f)$ is freely decomposable, then is $f$ freely decomposable?

(v) If $\mathcal{S F}_{n}(f)$ is freely decomposable, then is $\mathcal{F}_{n}(f)$ freely decomposable?

(vi) If $f$ is strongly freely decomposable, then is $\mathcal{F}_{2}(f)$ strongly freely decomposable?

(vii) If $f$ is strongly freely decomposable, then is $\mathcal{S F}_{2}(f)$ strongly freely decomposable?

(viii) If $\mathcal{F}_{2}(f)$ is strongly freely decomposable, then is $f$ strongly freely decomposable?

(ix) If $\mathcal{S F}_{n}(f)$ is strongly freely decomposable and $Y$ is not locally connected, then is $f$ strongly freely decomposable?

$(x)$ If $\mathcal{S F}_{n}(f)$ is strongly freely decomposable and $Y$ is not locally connected, then is $\mathcal{F}_{n}(f)$ strongly freely decomposable?

\section{JOINING MAPS}

We begin with an example that shows that there exists a joining map $f$ between continua such that the induced maps $\mathcal{F}_{2}(f)$ and $\mathcal{S F}_{2}(f)$ are not joining. 
ExAmPLE 7.1. Let $X=Y=[0,1]$ and let $f: X \rightarrow Y$ be the map given by:

$$
f(x)= \begin{cases}2 x+\frac{1}{2}, & \text { if } x \in\left[0, \frac{1}{4}\right] ; \\ -2 x+\frac{3}{2}, & \text { if } x \in\left[\frac{1}{4}, \frac{3}{4}\right] ; \\ 2 x-\frac{3}{2}, & \text { if } x \in\left[\frac{3}{4}, 1\right] .\end{cases}
$$

Then $f$ is a joining map. We see that $\mathcal{F}_{2}(f)$ and $\mathcal{S F}_{2}(f)$ are not joining. To this end, let

$$
\mathcal{B}=\left\{\left\{\frac{3}{4}, x\right\} \mid x \in\left[\frac{1}{4}, \frac{5}{8}\right]\right\} \cup\left\{\left\{\frac{1}{4}, x\right\} \mid x \in\left[\frac{3}{8}, \frac{3}{4}\right]\right\} .
$$

Since $\left\{\left\{\frac{3}{4}, x\right\} \mid x \in\left[\frac{1}{4}, \frac{5}{8}\right]\right\}$ and $\left\{\left\{\frac{1}{4}, x\right\} \mid x \in\left[\frac{3}{8}, \frac{3}{4}\right]\right\}$ are subcontinua of $\mathcal{F}_{2}(Y)$ having the point $\left\{\frac{1}{4}, \frac{3}{4}\right\}$ in common, it follows that $\mathcal{B}$ is a subcontinuum of $\mathcal{F}_{2}(Y)$. Observe that

$$
\begin{aligned}
\mathcal{F}_{2}(f)^{-1}(\mathcal{B})= & \left\{\left\{\frac{1}{8}, x\right\} \mid x \in\left[0, \frac{1}{16}\right]\right\} \\
& \cup\left\{\left\{\frac{1}{8}, x\right\} \mid x \in\left[\frac{7}{16}, \frac{5}{8}\right]\right\} \cup\left\{\left\{\frac{1}{8}, x\right\} \mid x \in\left[\frac{7}{8}, 1\right]\right\} \\
& \cup\left\{\left\{\frac{3}{8}, x\right\} \mid x \in\left[0, \frac{1}{16}\right]\right\} \cup\left\{\left\{\frac{3}{8}, x\right\} \mid x \in\left[\frac{7}{16}, \frac{5}{8}\right]\right\} \\
& \cup\left\{\left\{\frac{3}{8}, x\right\} \mid x \in\left[\frac{7}{8}, 1\right]\right\} \cup\left\{\left\{\frac{5}{8}, x\right\} \mid x \in\left[0, \frac{1}{8}\right]\right\} \\
& \cup\left\{\left\{\frac{5}{8}, x\right\} \mid x \in\left[\frac{3}{8}, \frac{9}{16}\right]\right\} \cup\left\{\left\{\frac{3}{8}, x\right\} \mid x \in\left[\frac{15}{16}, 1\right]\right\} \\
& \cup\left\{\left\{\frac{7}{8}, x\right\} \mid x \in\left[0, \frac{1}{8}\right]\right\} \cup\left\{\left\{\frac{7}{8}, x\right\} \mid x \in\left[\frac{3}{8}, \frac{9}{16}\right]\right\} \\
& \cup\left\{\left\{\frac{7}{8}, x\right\} \mid x \in\left[\frac{15}{16}, 1\right]\right\} .
\end{aligned}
$$

Let $\mathcal{C}=\left\{\left\{\frac{1}{8}, x\right\} \mid x \in\left[0, \frac{1}{16}\right]\right\}$ and $\mathcal{D}=\left\{\left\{\frac{7}{8}, x\right\} \mid x \in\left[\frac{15}{16}, 1\right]\right\}$. Note that $\mathcal{C}$ and $\mathcal{D}$ are components of $\mathcal{F}_{2}(f)^{-1}(\mathcal{B})$. Also, $\mathcal{F}_{2}(f)(\mathcal{C})=\left\{\left\{\frac{3}{4}, x\right\} \mid x \in\left[\frac{1}{2}, \frac{5}{8}\right]\right\}$ and $\mathcal{F}_{2}(f)(\mathcal{D})=\left\{\left\{\frac{1}{4}, x\right\} \mid x \in\left[\frac{3}{8}, \frac{1}{2}\right]\right\}$. Hence, $\mathcal{F}_{2}(f)(\mathcal{C}) \cap \mathcal{F}_{2}(f)(\mathcal{D})=\emptyset$. Thus, $\mathcal{F}_{2}(f)$ is not joining. To show that $\mathcal{S F}_{2}(f)$ is not joining, note that $q_{Y}^{2}(\mathcal{B})$ is a subcontinuum of $\mathcal{S F}_{2}(Y)$. Since $\mathcal{B} \cap \mathcal{F}_{1}(Y)=\emptyset$, by part $(g)$ of Proposition 3.1, $q_{X}^{2}(\mathcal{C})$ and $q_{X}^{2}(\mathcal{D})$ are components of $\mathcal{S F}_{2}(f)^{-1}\left(q_{Y}^{2}(\mathcal{B})\right.$ ). Suppose $\mathcal{S F}_{2}(f)\left(q_{X}^{2}(\mathcal{C})\right) \cap \mathcal{S F}_{2}(f)\left(q_{X}^{2}(\mathcal{D})\right) \neq \emptyset$. Then, since $(*)$ commutes, $q_{Y}^{2}\left(\mathcal{F}_{2}(f)(\mathcal{C})\right) \cap q_{Y}^{2}\left(\mathcal{F}_{2}(f)(\mathcal{D})\right) \neq \emptyset$. Since $\mathcal{B} \cap \mathcal{F}_{1}(Y)=\emptyset$, by part $(b)$ of Proposition 3.1, $\mathcal{F}_{2}(f)(\mathcal{C}) \cap \mathcal{F}_{2}(f)(\mathcal{D}) \neq \emptyset$, a contradiction. Hence, $\mathcal{S F}_{2}(f)\left(q_{X}^{2}(\mathcal{C})\right) \cap \mathcal{S F}_{2}(f)\left(q_{X}^{2}(\mathcal{D})\right)=\emptyset$. Thus, $\mathcal{S F}_{2}(f)$ is not joining. 
TheOREM 7.2. Let $X$ and $Y$ be continua, let $n$ be an integer greater than or equal to 2 , and let $f: X \rightarrow Y$ be a surjective map. Consider the following statements:

(1) $f$ is joining;

(2) $\mathcal{F}_{n}(f)$ is joining;

(3) $\mathcal{S F}_{n}(f)$ is joining.

Then (2) implies (1) and (3), (3) implies (1), and (1) does not imply either (2) or (3) for $n=2$.

Proof. It follows from Theorem 3.2 that (2) implies (3). Suppose $\mathcal{S F}_{n}(f)$ is joining, we prove that $f$ is joining. To this end, let $B$ be a subcontinuum of $Y$. If $B=Y$, then $f^{-1}(B)$ only has one component, namely $X$, and $f(X)=B$. Suppose $B \neq Y$ and let $C$ and $D$ be two components of $f^{-1}(B)$. Since $B \neq Y$, there exist $n-1$ distinct points $y_{1}, \ldots, y_{n-1}$ in $Y \backslash B$. Let

$$
\mathcal{K}=\left\{\left\{y_{1}, \ldots, y_{n-1}\right\} \cup\{b\} \mid b \in B\right\} .
$$

Then $\mathcal{K}$ is a subcontinuum of $\mathcal{F}_{n}(Y)$ and $\mathcal{K} \cap \mathcal{F}_{1}(Y)=\emptyset$. Hence, $q_{Y}^{n}(\mathcal{K})$ is a subcontinuum of $\mathcal{S F}_{n}(Y) \backslash\left\{F_{Y}^{n}\right\}$. For each $j \in\{1, \ldots, n-1\}$, let $C_{j}$ be a component of $f^{-1}\left(y_{j}\right)$. Let $\mathcal{C}=\left\langle C_{1}, \ldots, C_{n-1}, C\right\rangle_{n}$ and $\mathcal{D}=\left\langle C_{1}, \ldots, C_{n-1}, D\right\rangle_{n}$. Then by Lemma $3.6, \mathcal{C}$ and $\mathcal{D}$ are components of $\mathcal{F}_{n}(f)^{-1}(\mathcal{K})$. By part $(g)$ of Proposition 3.1, $q_{X}^{n}(\mathcal{C})$ and $q_{X}^{n}(\mathcal{D})$ are components of $\mathcal{S F}_{n}(f)^{-1}\left(q_{Y}^{n}(\mathcal{K})\right)$. Since $\mathcal{S} \mathcal{F}_{n}(f)$ is joining, we have that $\mathcal{S F}_{n}(f)\left(q_{X}^{n}(\mathcal{C})\right) \cap \mathcal{S F}_{n}(f)\left(q_{X}^{n}(\mathcal{D})\right) \neq \emptyset$. Hence, since $(*)$ is commutative, $q_{X}^{n}\left(\mathcal{F}_{n}(f)(\mathcal{C})\right) \cap q_{X}^{n}\left(\mathcal{F}_{n}(f)(\mathcal{D})\right) \neq \emptyset$. Thus, by part $(b)$ of Proposition 3.1, $\mathcal{F}_{n}(f)(\mathcal{C}) \cap \mathcal{F}_{n}(f)(\mathcal{D}) \neq \emptyset$. Let $E \in \mathcal{F}_{n}(f)(\mathcal{C}) \cap \mathcal{F}_{n}(f)(\mathcal{D})$. Then there exist $A \in \mathcal{C}$ and $A^{\prime} \in \mathcal{D}$ such that $\mathcal{F}_{n}(f)(A)=\mathcal{F}_{n}(f)\left(A^{\prime}\right)=E$. Without loss of generality, we may assume that $A=\left\{c_{1}, \ldots, c_{n-1}, c\right\}$ and $A^{\prime}=\left\{c_{1}^{\prime}, \ldots, c_{n-1}^{\prime}, d\right\}$, where, for each $j \in\{1, \ldots, n-1\}, c_{j}$ and $c_{j}^{\prime}$ belong to $C_{j}, c \in C$ and $d \in D$. Since $\mathcal{F}_{n}(f)(A)=\mathcal{F}_{n}(f)\left(A^{\prime}\right)$, we obtain that $\left\{f\left(c_{1}\right), \ldots, f\left(c_{n-1}\right), f(c)\right\}=\left\{f\left(c_{1}^{\prime}\right), \ldots, f\left(c_{n-1}^{\prime}\right), f(d)\right\}$. This implies that $f(c)=f(d)$ and $f(C) \cap f(D) \neq \emptyset$. Therefore, $f$ is joining.

Since (2) implies (3) and (3) implies (1), we have that (2) implies (1). The fact that (1) does not imply either (2) or (3), when $n=2$, follows from Example 7.1.

Questions 7.3. Let $X$ and $Y$ be continua, let $n$ be an integer greater than or equal to 2 , and let $f: X \rightarrow Y$ be a surjective map.

(i) If $f$ is joining, then is $\mathcal{S F}_{n}(f)$ joining?

(ii) If $\mathcal{S F}_{n}(f)$ is joining, then is $\mathcal{F}_{n}(f)$ joining?

REMARK 7.4. Note that, by Example 7.1, the answer to Question $7.3(i)$ is negative when $n=2$. 


\section{ReFinable AND MONOtONICALly REFinABle MAPS}

Let $X$ be a continuum and let $n$ be an integer greater than or equal to 2. We follow Sam B. Nadler, Jr. ([16], compare with [12, p. 149]) to define a metric on $\mathcal{S F}_{n}(X)$. Let

$$
\Im_{n}(X)=\left\{\mathcal{F}_{1}(X) \cup\{A\} \mid A \in \mathcal{F}_{n}(X)\right\} .
$$

Note that $\Im_{n}(X) \subset \mathcal{C}_{2}\left(\mathcal{F}_{n}(X)\right)$. Let $G_{n}: \mathcal{S} \mathcal{F}_{n}(X) \rightarrow \Im_{n}(X)$ be given by

$$
G_{n}(\chi)=\mathcal{F}_{1}(X) \cup\left(q_{X}^{n}\right)^{-1}(\chi) .
$$

Then $G_{n}$ is a homeomorphism. Next, define

$$
\rho_{X}^{n}: \mathcal{S} \mathcal{F}_{n}(X) \times \mathcal{S F}_{n}(X) \rightarrow[0, \infty)
$$

by

$$
\rho_{X}^{n}\left(\chi_{1}, \chi_{2}\right)=\mathcal{H}_{X}^{2}\left(G_{n}\left(\chi_{1}\right), G_{n}\left(\chi_{2}\right)\right),
$$

where $\mathcal{H}_{X}^{2}$ is the Hausdorff metric on $\mathcal{C}_{2}\left(\mathcal{F}_{n}(X)\right)$ induced by the Hausdorff metric $\mathcal{H}_{X}$ on $\mathcal{F}_{n}(X)$. Then $\rho_{X}^{n}$ is a metric. As a consequence of $[16,(2.3)]$, with our terminology, we obtain:

LEMMA 8.1. Let $X$ and $Y$ be continua, let $n$ be an integer greater than or equal to 2 , and let $\varepsilon>0$. If $f: X \rightarrow Y$ is a surjective map such that $\mathcal{F}_{n}(f)$ is an $\varepsilon$-map, then $\mathcal{S F}_{n}(f)$ is an $\varepsilon$-map.

Lemma 8.2. Let $X$ and $Y$ be continua, let $n$ be an integer greater than or equal to 2 , and let $\varepsilon>0$. If $f: X \rightarrow Y$ is a surjective map, then the following are equivalent:

(1) $f$ is an $\varepsilon$-map;

(2) $\mathcal{F}_{n}(f)$ is an $\varepsilon$-map;

(3) $\mathcal{S F}_{n}(f)$ is an $\varepsilon$-map.

Proof. Suppose $f$ is an $\varepsilon$-map, we show that $\mathcal{F}_{n}(f)$ is an $\varepsilon$-map. Since $f$ is surjective, by Lemma $3.4, \mathcal{F}_{n}(f)$ is surjective. Let $B \in \mathcal{F}_{n}(Y)$ and let $A_{1}$ and $A_{2}$ be two elements of $\mathcal{F}_{n}(f)^{-1}(B)$. Since $\mathcal{F}_{n}(f)\left(A_{1}\right)=\mathcal{F}_{n}(f)\left(A_{2}\right)$, for each $a_{1} \in A_{1}$ there exists $a_{2} \in A_{2}$ such that $f\left(a_{1}\right)=f\left(a_{2}\right)$. Since $f$ is an $\varepsilon$-map, $d\left(a_{1}, a_{2}\right)<\varepsilon$. Hence, $A_{1} \subset \mathcal{V}_{\varepsilon}\left(A_{2}\right)$. Similarly, $A_{2} \subset \mathcal{V}_{\varepsilon}\left(A_{1}\right)$. Thus, $\mathcal{H}_{X}\left(A_{1}, A_{2}\right)<\varepsilon$. Therefore, $\mathcal{F}_{n}(f)$ is an $\varepsilon$-map.

Assume that $\mathcal{F}_{n}(f)$ is an $\varepsilon$-map, we prove that $f$ is an $\varepsilon$-map. By Lemma 3.4, $f$ is surjective. Let $y \in Y$ and let $x_{1}$ and $x_{2}$ be two points in $f^{-1}(y)$. Then $\mathcal{F}_{n}(f)\left(\left\{x_{1}\right\}\right)=\mathcal{F}_{n}(f)\left(\left\{x_{2}\right\}\right)$. Since $\mathcal{F}_{n}(f)$ is an $\varepsilon$-map, $\mathcal{H}_{X}\left(\left\{x_{1}\right\},\left\{x_{2}\right\}\right)<\varepsilon$. Thus, $d\left(x_{1}, x_{2}\right)<\varepsilon$. Therefore, $f$ is an $\varepsilon$-map.

It follows from Lemma 8.1 that if $\mathcal{F}_{n}(f)$ is an $\varepsilon$-map, then $\mathcal{S F}_{n}(f)$ is an $\varepsilon$-map.

Suppose $\mathcal{S F}_{n}(f)$ is an $\varepsilon$-map, we show that $\mathcal{F}_{n}(f)$ is an $\varepsilon$-map. By Lemma $3.4, \mathcal{F}_{n}(f)$ is surjective. Let $B \in \mathcal{F}_{n}(Y)$ and let $A_{1}$ and $A_{2}$ be two 
elements of $\mathcal{F}_{n}(f)^{-1}(B)$. Since $\mathcal{F}_{n}(f)\left(A_{1}\right)=\mathcal{F}_{n}(f)\left(A_{2}\right)$ and $(*)$ is commutative, we have that $\mathcal{S F}_{n}(f)\left(q_{X}^{n}\left(A_{1}\right)\right)=\mathcal{S F}_{n}(f)\left(q_{X}^{n}\left(A_{2}\right)\right)$. Since $\mathcal{S F}_{n}(f)$ is an $\varepsilon$ map, $\rho_{X}^{n}\left(q_{X}^{n}\left(A_{1}\right), q_{X}^{n}\left(A_{2}\right)\right)<\varepsilon$. Hence, $\mathcal{H}_{X}^{2}\left(\mathcal{F}_{1}(X) \cup\left\{A_{1}\right\}, \mathcal{F}_{1}(X) \cup\left\{A_{2}\right\}\right)<\varepsilon$. Thus, $\mathcal{H}_{X}\left(A_{1}, A_{2}\right)<\varepsilon$. Therefore, $\mathcal{F}_{n}(f)$ is an $\varepsilon$-map.

LEMMA 8.3. Let $X$ and $Y$ be continua, let $n$ be an integer greater than or equal to 2 , and let $\varepsilon>0$. Let $f, g: X \rightarrow Y$ be surjective maps. Consider the following statements:

(1) For each $x \in X, d(f(x), g(x))<\varepsilon$;

(2) For each $A \in \mathcal{F}_{n}(X), \mathcal{H}_{Y}\left(\mathcal{F}_{n}(f)(A), \mathcal{F}_{n}(g)(A)\right)<\varepsilon$;

(3) For each $\chi \in \mathcal{S F}_{n}(X), \rho_{Y}^{n}\left(\mathcal{S F}_{n}(f)(\chi), \mathcal{S F}_{n}(g)(\chi)\right)<\varepsilon$;

(4) For each $A \in \mathcal{F}_{n}(X), \mathcal{H}_{Y}\left(\mathcal{F}_{n}(f)(A), \mathcal{F}_{n}(g)(A)\right) \leq \varepsilon$.

Then (1) and (2) are equivalent, (2) implies (3) and (3) implies (4).

Proof. Suppose (1) holds, we prove (2) holds. Let $A \in \mathcal{F}_{n}(X)$. Then for each $a \in A, d(f(a), g(a))<\varepsilon$. Hence, $\mathcal{F}_{n}(f)(A) \subset \mathcal{V}_{\varepsilon}\left(\mathcal{F}_{n}(g)(A)\right)$ and $\mathcal{F}_{n}(g)(A) \subset \mathcal{V}_{\varepsilon}\left(\mathcal{F}_{n}(f)(A)\right)$. Therefore, $\mathcal{H}_{Y}\left(\mathcal{F}_{n}(f)(A), \mathcal{F}_{n}(g)(A)\right)<\varepsilon$.

Assume (2) holds, we show that (1) holds. Let $x \in X$. Then $\mathcal{H}_{Y}\left(\mathcal{F}_{n}(f)(\{x\}), \mathcal{F}_{n}(g)(\{x\})\right)<\varepsilon$. Since $\mathcal{H}_{Y}\left(\mathcal{F}_{n}(f)(\{x\}), \mathcal{F}_{n}(g)(\{x\})\right)=$ $d(f(x), g(x))$, we obtain that $d(f(x), g(x))<\varepsilon$.

Suppose (2) holds, we prove (3) holds. Let $\chi \in \mathcal{S F}_{n}(X)$. If $\chi=F_{X}^{n}$, then $\mathcal{S F}_{n}(f)(\chi)=F_{Y}^{n}=\mathcal{S F}_{n}(g)(\chi)$ and $\rho_{Y}^{n}\left(\mathcal{S F}_{n}(f)(\chi), \mathcal{S F}_{n}(g)(\chi)\right)=0<\varepsilon$. Assume $\chi \in \mathcal{S} \mathcal{F}_{n}(X) \backslash\left\{F_{X}^{n}\right\}$. Then, since $(*)$ is commutative, we obtain

$$
\begin{gathered}
\rho_{Y}^{n}\left(\mathcal{S F}_{n}(f)(\chi), \mathcal{S F}_{n}(g)(\chi)\right)= \\
\mathcal{H}_{Y}^{2}\left(\mathcal{F}_{1}(Y) \cup\left(q_{Y}^{n}\right)^{-1}\left(\mathcal{S F}_{n}(f)(\chi)\right), \mathcal{F}_{1}(Y) \cup\left(q_{Y}^{n}\right)^{-1}\left(\mathcal{S F}_{n}(g)(\chi)\right)\right)= \\
\mathcal{H}_{Y}^{2}\left(\mathcal{F}_{1}(Y) \cup\left\{\mathcal{F}_{n}(f)\left(\left(q_{X}^{n}\right)^{-1}(\chi)\right)\right\}, \mathcal{F}_{1}(Y) \cup\left\{\mathcal{F}_{n}(g)\left(\left(q_{X}^{n}\right)^{-1}(\chi)\right)\right\}\right)<\varepsilon .
\end{gathered}
$$

Suppose (3) holds, we show that (4) holds. Let $A \in \mathcal{F}_{n}(X)$. Assume first that $A \in \mathcal{F}_{n}(X) \backslash \mathcal{F}_{1}(X)$. Then $q_{X}^{n}(A) \in \mathcal{S} \mathcal{F}_{n}(X) \backslash\left\{F_{X}^{n}\right\}$. Hence, $\rho_{Y}^{n}\left(\mathcal{S F}_{n}(f)\left(q_{X}^{n}(A)\right), \mathcal{S F}_{n}(g)\left(q_{X}^{n}(A)\right)\right)<\varepsilon$; i.e.

$$
\begin{gathered}
\mathcal{H}_{Y}^{2}\left(\mathcal{F}_{1}(Y) \cup\left(q_{Y}^{n}\right)^{-1}\left(\mathcal{S F}_{n}(f)\left(q_{X}^{n}(A)\right)\right), \mathcal{F}_{1}(Y) \cup\left(q_{Y}^{n}\right)^{-1}\left(\mathcal{S} \mathcal{F}_{n}(g)\left(q_{X}^{n}(A)\right)\right)\right)= \\
\mathcal{H}_{Y}^{2}\left(\mathcal{F}_{1}(Y) \cup\left\{\mathcal{F}_{n}(f)\left(\left(q_{X}^{n}\right)^{-1}\left(q_{X}^{n}(A)\right)\right)\right\}, \mathcal{F}_{1}(Y) \cup\left\{\mathcal{F}_{n}(g)\left(\left(q_{X}^{n}\right)^{-1}\left(q_{X}^{n}(A)\right)\right)\right\}\right)= \\
\mathcal{H}_{Y}^{2}\left(\mathcal{F}_{1}(Y) \cup\left\{\mathcal{F}_{n}(f)(A)\right\}, \mathcal{F}_{1}(Y) \cup\left\{\mathcal{F}_{n}(g)(A)\right\}\right)= \\
\mathcal{H}_{Y}\left(\mathcal{F}_{n}(f)(A), \mathcal{F}_{n}(g)(A)\right)<\varepsilon .
\end{gathered}
$$

Now suppose $A \in \mathcal{F}_{1}(X)$. Then $A=\{a\}$. Let $\left\{a_{m}\right\}_{m=1}^{\infty}$ be a sequence of points of $X$ converging to $a$ such that $a_{m} \neq a$ for any positive integer $m$. Then $\left\{a, a_{m}\right\} \in \mathcal{F}_{n}(X) \backslash \mathcal{F}_{1}(X)$. By the previous argument, $\mathcal{H}_{Y}\left(\mathcal{F}_{n}(f)\left(\left\{a, a_{m}\right\}\right), \mathcal{F}_{n}(g)\left(\left\{a, a_{m}\right\}\right)\right)<\varepsilon$. Hence, since $\left\{\left\{a, a_{m}\right\}\right\}_{m=1}^{\infty}$ converges to $\{a\}$, by the continuity of $\mathcal{F}_{n}(f), \mathcal{F}_{n}(g)$ and $\mathcal{H}_{Y}$, we have that $\mathcal{H}_{Y}\left(\mathcal{F}_{n}(f)(\{a\}), \mathcal{F}_{n}(g)(\{a\})\right) \leq \varepsilon$. 
Theorem 8.4. Let $X$ and $Y$ be continua, let $n$ be an integer greater than or equal to 2, and let $f: X \rightarrow Y$ be a surjective map. Consider the following statements:

(1) $f$ is refinable;

(2) $\mathcal{F}_{n}(f)$ is refinable;

(3) $\mathcal{S F}_{n}(f)$ is refinable.

Then (1) implies (2) and (3).

Proof. The fact that (1) implies (2) follows from [9, Theorem 3.49]. Suppose $f$ is refinable, we show that $\mathcal{S F}_{n}(f)$ is refinable. Let $\varepsilon>0$. Since $f$ is refinable, there exists an $\varepsilon$-map $g: X \rightarrow Y$ such that for every $x \in X$, $d(f(x), g(x))<\varepsilon$. Then, by Lemma $8.2, \mathcal{S F}_{n}(g)$ is an $\varepsilon$-map and, by Lemma 8.3, for each $\chi \in \mathcal{S F}_{n}(X), \rho_{Y}^{n}\left(\mathcal{S F}_{n}(f)(\chi), \mathcal{S F}_{n}(g)(\chi)\right)<\varepsilon$. Therefore, $\mathcal{S F}_{n}(f)$ is refinable.

TheOREM 8.5. Let $X$ and $Y$ be continua, let $n$ be an integer greater than or equal to 2, and let $f: X \rightarrow Y$ be a surjective map. Consider the following statements:

(1) $f$ is monotonically refinable;

(2) $\mathcal{F}_{n}(f)$ is monotonically refinable;

(3) $\mathcal{S F}_{n}(f)$ is monotonically refinable.

Then (1) implies (2) and (3).

Proof. Suppose $f$ is monotonically refinable, we show that $\mathcal{F}_{n}(f)$ and $\mathcal{S F}_{n}(f)$ are monotonically refinable. Let $\varepsilon>0$. Since $f$ is monotonically refinable, there exists a monotone $\varepsilon$-map $g: X \rightarrow Y$ such that for every $x \in X$, $d(f(x), g(x))<\varepsilon$. By Lemma 8.2, $\mathcal{F}_{n}(g)$ and $\mathcal{S F}_{n}(g)$ are $\varepsilon$-maps. By $[2$, Theorem 4.1], $\mathcal{F}_{n}(g)$ and $\mathcal{S F}_{n}(g)$ are monotone maps. Now, by Lemma 8.3, we have that for each $A \in \mathcal{F}_{n}(X), \mathcal{H}_{Y}\left(\mathcal{F}_{n}(f)(A), \mathcal{F}_{n}(g)(A)\right)<\varepsilon$; and for each $\chi \in \mathcal{S F}_{n}(X), \rho_{Y}^{n}\left(\mathcal{S F}_{n}(f)(\chi), \mathcal{S F}_{n}(g)(\chi)\right)<\varepsilon$. Therefore, $\mathcal{F}_{n}(f)$ and $\mathcal{S F}_{n}(f)$ are monotonically refinable maps.

Questions 8.6. Let $X$ and $Y$ be continua, let $n$ be an integer greater than or equal to 2 , and let $f: X \rightarrow Y$ be a surjective map.

(i) If $\mathcal{F}_{n}(f)$ is (monotonically) refinable, then is $f$ (monotonically) refinable?

(ii) If $\mathcal{F}_{n}(f)$ is (monotonically) refinable, then is $\mathcal{S F}_{n}(f)$ (monotonically) refinable?

(iii) If $\mathcal{S F}_{n}(f)$ is (monotonically) refinable, then is $f$ (monotonically) refinable?

(iv) If $\mathcal{S F}_{n}(f)$ is (monotonically) refinable, then is $\mathcal{F}_{n}(f)$ (monotonically) refinable? 


\section{SEMi-CONFLUent MAPS}

Theorem 9.1. Let $X$ and $Y$ be continua, let $n$ be an integer greater than or equal to 2, and let $f: X \rightarrow Y$ be a surjective map. Consider the following statements:

(1) $f$ is semi-confluent;

(2) $\mathcal{F}_{n}(f)$ is semi-confluent;

(3) $\mathcal{S F}_{n}(f)$ is semi-confluent.

Then (2) implies (1) and (3), (3) implies (1); and (1) does not imply either (2) or (3) for $n=2$.

Proof. Suppose $\mathcal{S F}_{n}(f)$ is semi-confluent, we show that $f$ is semiconfluent. Let $B$ be a subcontinuum of $Y$. If $B=Y$, then $f^{-1}(B)$ has only one component, namely $X$ and $f(X)=B$. Suppose $B \neq Y$ and let $C$ and $D$ be two components of $f^{-1}(B)$. Let $y_{1}, \ldots, y_{n-1}$ be $n-1$ distinct elements of $Y \backslash B$. Let

$$
\mathcal{K}=\left\{\left\{y_{1}, \ldots, y_{n-1}\right\} \cup\{b\} \mid b \in B\right\} .
$$

Note that $\mathcal{K}$ is a subcontinuum of $\mathcal{F}_{n}(Y) \backslash \mathcal{F}_{1}(Y)$. Hence, $q_{Y}^{n}(\mathcal{K})$ is a subcontinuum of $\mathcal{S F}_{n}(Y) \backslash\left\{F_{Y}^{n}\right\}$. For each $j \in\{1, \ldots, n-1\}$, let $C_{j}$ be a component of $f^{-1}\left(y_{j}\right)$. Let $\mathcal{C}=\left\langle C_{1}, \ldots, C_{n-1}, C\right\rangle_{n}$ and $\mathcal{D}=\left\langle C_{1}, \ldots, C_{n-1}, D\right\rangle_{n}$. Then by Lemma 3.6, $\mathcal{C}$ and $\mathcal{D}$ are components of $\mathcal{F}_{n}(f)^{-1}(\mathcal{K})$. By part $(g)$ of Proposition 3.1, $q_{X}^{n}(\mathcal{C})$ and $q_{X}^{n}(\mathcal{D})$ are components of $\mathcal{S} \mathcal{F}_{n}(f)^{-1}\left(q_{Y}^{n}(\mathcal{K})\right)$. Since $\mathcal{S F}_{n}(f)$ is semi-confluent, we have that either $\mathcal{S F}_{n}(f)\left(q_{X}^{n}(\mathcal{C})\right) \subset$ $\mathcal{S F}_{n}(f)\left(q_{X}^{n}(\mathcal{D})\right)$ or $\mathcal{S F}_{n}(f)\left(q_{X}^{n}(\mathcal{D})\right) \subset \mathcal{S F}_{n}(f)\left(q_{X}^{n}(\mathcal{C})\right)$. Hence, since $(*)$ is commutative, we have that either $q_{Y}^{n}\left(\mathcal{F}_{n}(f)(\mathcal{C})\right) \subset q_{Y}^{n}\left(\mathcal{F}_{n}(f)(\mathcal{D})\right)$ or $q_{Y}^{n}\left(\mathcal{F}_{n}(f)(\mathcal{D})\right) \subset q_{Y}^{n}\left(\mathcal{F}_{n}(f)(\mathcal{C})\right)$. Thus, by part $(b)$ of Proposition 3.1, either $\mathcal{F}_{n}(f)(\mathcal{C}) \subset \mathcal{F}_{n}(f)(\mathcal{D})$ or $\mathcal{F}_{n}(f)(\mathcal{D}) \subset \mathcal{F}_{n}(f)(\mathcal{C})$. Assume $\mathcal{F}_{n}(f)(\mathcal{C}) \subset$ $\mathcal{F}_{n}(f)(\mathcal{D})$, we see that $f(C) \subset f(D)$. Let $c \in C$ and, for each $j \in\{1, \ldots, n-$ $1\}$, let $c_{j} \in C_{j}$. Then $\left\{c_{1}, \ldots, c_{n-1}, c\right\} \in \mathcal{C}$. Since $\mathcal{F}_{n}(f)\left(\left\{c_{1}, \ldots, c_{n-1}, c\right\}\right) \in$ $\mathcal{F}_{n}(f)(\mathcal{D})$, there exist $d \in D$ and $c_{j}^{\prime} \in C_{j}$, where $j \in\{1, \ldots, n-1\}$, such that

$$
\mathcal{F}_{n}(f)\left(\left\{c_{1}, \ldots, c_{n-1}, c\right\}\right)=\mathcal{F}_{n}(f)\left(\left\{c_{1}^{\prime}, \ldots, c_{n-1}^{\prime}, d\right\}\right) .
$$

Hence, $f\left(\left\{c_{1}, \ldots, c_{n-1}, c\right\}\right)=f\left(\left\{c_{1}^{\prime}, \ldots, c_{n-1}^{\prime}, d\right\}\right)$. Thus, $f(c)=f(d)$ and $f(C) \subset f(D)$. Similarly, if $\mathcal{F}_{n}(f)(\mathcal{D}) \subset \mathcal{F}_{n}(f)(\mathcal{C})$, then $f(D) \subset f(C)$. Therefore, $f$ is semi-confluent.

It follows from Theorem 3.2 that (2) implies (3). Since (2) implies (3) and (3) implies (1), we have that (2) implies (1). The fact that (1) does not imply (2), when $n=2$ follows from [9, Example 3.18]. By [2, Example 10.7], we have that (1) does not imply (3) when $n=2$.

Question 9.1. Let $X$ and $Y$ be continua, let $n$ be an integer greater than or equal to 2 , and let $f: X \rightarrow Y$ be a surjective map. If $\mathcal{S F}_{n}(f)$ is semi-confluent, then is $\mathcal{F}_{n}(f)$ semi-confluent? 


\section{SEMI-OPEN MAPS}

TheOREM 10.1. Let $X$ and $Y$ be continua and let $n$ be an integer greater than or equal to 2. If $f: X \rightarrow Y$ is a surjective map, then the following are equivalent:

(1) $f$ is semi-open;

(2) $\mathcal{F}_{n}(f)$ is semi-open;

(3) $\mathcal{S F}_{n}(f)$ is semi-open.

Proof. Suppose $f$ is semi-open, we show that $\mathcal{F}_{n}(f)$ is semi-open. Let $\mathcal{U}$ be an open subset of $\mathcal{F}_{n}(X)$ and let $A_{0} \in \mathcal{U}$. Then there exist open subsets $U_{1}, \ldots, U_{k}$ of $X$ such that $A_{0} \in\left\langle U_{1}, \ldots, U_{k}\right\rangle_{n} \subset \mathcal{U}$. Since $f$ is semi-open, for each $j \in\{1, \ldots, k\}, \operatorname{Int}_{Y}\left(f\left(U_{j}\right)\right) \neq \emptyset$. Thus, $\left\langle\operatorname{Int}_{Y}\left(f\left(U_{1}\right)\right), \ldots, \operatorname{Int}_{Y}\left(f\left(U_{k}\right)\right)\right\rangle_{n}$ is an open subset of $\mathcal{F}_{n}(Y)$. We claim that $\left\langle\operatorname{Int}_{Y}\left(f\left(U_{1}\right)\right), \ldots, \operatorname{Int}_{Y}\left(f\left(U_{k}\right)\right)\right\rangle_{n} \subset \mathcal{F}_{n}(f)(\mathcal{U})$. To see this, let $B \in$ $\left\langle\operatorname{Int}_{Y}\left(f\left(U_{1}\right)\right), \ldots, \operatorname{Int}_{Y}\left(f\left(U_{k}\right)\right)\right\rangle_{n}$. For every $j \in\{1, \ldots, k\}$, let $B_{j}=B \cap$ $\operatorname{Int}_{Y}\left(f\left(U_{j}\right)\right)$. Then there exists $A_{j} \subset U_{j}$ such that $A_{j}$ and $B_{j}$ both have the same cardinality and $f\left(A_{j}\right)=B_{j}$. Let $A=\bigcup_{j=1}^{k} A_{j}$. Then $A \in\left\langle U_{1}, \ldots, U_{k}\right\rangle_{n}$ and $\mathcal{F}_{n}(f)(A)=B$. Hence, $B \in \mathcal{F}_{n}(f)\left(\left\langle U_{1}, \ldots, U_{k}\right\rangle_{n}\right) \subset \mathcal{F}_{n}(f)(\mathcal{U})$. Thus, $\operatorname{Int}_{\mathcal{F}_{n}(Y)}\left(\mathcal{F}_{n}(f)(\mathcal{U})\right) \neq \emptyset$. Therefore, $\mathcal{F}_{n}(f)$ is a semi-open map.

Assume $\mathcal{F}_{n}(f)$ is semi-open, we prove that $f$ is semi-open. Let $U$ be an open subset of $X$. Since $\mathcal{F}_{n}(f)$ is semi-open, $\operatorname{Int}_{\mathcal{F}_{n}(Y)}\left(\mathcal{F}_{n}(f)\left(\langle U\rangle_{n}\right)\right) \neq \emptyset$. We consider two cases.

First, suppose that $\operatorname{Int}_{\mathcal{F}_{n}(Y)}\left(\mathcal{F}_{n}(f)\left(\langle U\rangle_{n}\right)\right) \cap \mathcal{F}_{1}(Y) \neq \emptyset$. Let $\left\{y_{0}\right\} \in$ $\operatorname{Int}_{\mathcal{F}_{n}(Y)}\left(\mathcal{F}_{n}(f)\left(\langle U\rangle_{n}\right)\right) \cap \mathcal{F}_{1}(Y)$. Then there exist open subsets $V_{1}, \ldots, V_{m}$ of $Y$ such that $\left\{y_{0}\right\} \in\left\langle V_{1}, \ldots, V_{m}\right\rangle_{n} \subset \operatorname{Int}_{\mathcal{F}_{n}(Y)}\left(\mathcal{F}_{n}(f)\left(\langle U\rangle_{n}\right)\right)$. Note that $y_{0} \in \bigcap_{j=1}^{m} V_{j}$. We claim that $\bigcap_{j=1}^{m} V_{j} \subset f(U)$. Let $y \in \bigcap_{j=1}^{m} V_{j}$. Then $\{y\} \in$ $\left\langle V_{1}, \ldots, V_{m}\right\rangle_{n}$, and there exists $A_{y} \in\langle U\rangle_{n}$ such that $\mathcal{F}_{n}(f)\left(A_{y}\right)=\{y\}$. This means that $A_{y} \subset U$ and $f\left(A_{y}\right)=\{y\}$. Thus, $y \in f(U)$ and $\bigcap_{j=1}^{m} V_{j} \subset f(U)$.

Now, assume that $\operatorname{Int}_{\mathcal{F}_{n}(Y)}\left(\mathcal{F}_{n}(f)\left(\langle U\rangle_{n}\right)\right) \cap \mathcal{F}_{1}(Y)=\emptyset$. Let $\ell=\min \{j \in$ $\left.\{2, \ldots, n\} \mid \operatorname{Int}_{\mathcal{F}_{n}(Y)}\left(\mathcal{F}_{n}(f)\left(\langle U\rangle_{n}\right)\right) \cap \mathcal{F}_{j}(Y) \neq \emptyset\right\}$. Let

$$
\left\{y_{1}, \ldots, y_{\ell}\right\} \in \operatorname{Int}_{\mathcal{F}_{n}(Y)}\left(\mathcal{F}_{n}(f)\left(\langle U\rangle_{n}\right)\right) \cap \mathcal{F}_{\ell}(Y) .
$$

Then there exist open subsets $W_{1}, \ldots, W_{r}$ of $Y$ such that $\left\{y_{1}, \ldots, y_{\ell}\right\} \in$ $\left\langle W_{1}, \ldots, W_{r}\right\rangle_{n} \subset \operatorname{Int}_{\mathcal{F}_{n}(Y)}\left(\mathcal{F}_{n}(f)\left(\langle U\rangle_{n}\right)\right)$. For each $j \in\{1, \ldots, \ell\}$, let $Z_{j}=\bigcap\left\{W_{k} \in\left\{W_{1}, \ldots, W_{r}\right\} \mid y_{j} \in W_{k}\right\}$. Note that each $Z_{j}$ is an open subset of $Y$, and $\left\{y_{1}, \ldots, y_{\ell}\right\} \in\left\langle Z_{1}, \ldots, Z_{\ell}\right\rangle_{n} \subset\left\langle W_{1}, \ldots, W_{r}\right\rangle_{n}$. Let $Z=\bigcup_{j=1}^{\ell} Z_{j}$. We claim that $Z \subset f(U)$. Let $z \in Z$. Without loss of generality, we assume that $z \in Z_{1}$. For every $j \in\{2, \ldots, \ell\}$, let $z_{j} \in Z_{j}$. Then $\left\{z, z_{2}, \ldots, z_{\ell}\right\} \in\left\langle Z_{1}, \ldots, Z_{\ell}\right\rangle_{n} \subset \operatorname{Int}_{\mathcal{F}_{n}(Y)}\left(\mathcal{F}_{n}(f)\left(\langle U\rangle_{n}\right)\right)$. Hence, there exists $A_{z} \in\langle U\rangle_{n}$ such that $\mathcal{F}_{n}(f)\left(A_{z}\right)=\left\{z, z_{2}, \ldots, z_{\ell}\right\}$. This implies that $A_{z} \subset U$ and $f\left(A_{z}\right)=\left\{z, z_{2}, \ldots, z_{\ell}\right\}$. Thus, there exists $a \in A_{z}$ such that $f(a)=z$. Hence, $z \in f(U)$ and $Z \subset f(U)$. Therefore, $f$ is a semi-open map. 
The fact that (2) and (3) are equivalent follows from Theorem 3.3.

\section{Simple MAPS}

TheOREM 11.1. Let $X$ and $Y$ be continua, let $n$ be an integer greater than or equal to 2 , and let $f: X \rightarrow Y$ be a surjective map. If $\mathcal{F}_{n}(f)$ is a simple map, then $f$ is a homeomorphism.

Proof. If $f$ is not one-to-one, then there exist two distinct points $x_{1}$ and $x_{2}$ in $X$ such that $f\left(x_{1}\right)=f\left(x_{2}\right)$. Let $f\left(x_{1}\right)=y$. Then $\left\{x_{1}\right\},\left\{x_{2}\right\}$ and $\left\{x_{1}, x_{2}\right\}$ are 3 distinct points in $\mathcal{F}_{n}(f)^{-1}(\{y\})$. Therefore, $\mathcal{F}_{n}(f)$ is not simple.

The following example shows that there exists a simple map $f$ between locally connected continua such that neither $\mathcal{F}_{n}(f)$ nor $\mathcal{S F}_{n}(f)$ is a simple map.

EXAMPLE 11.2. Let $f: \mathcal{S}^{1} \rightarrow \mathcal{S}^{1}$ be given by $f(z)=z^{2}$. Then $f$ is a simple map. Let $z$ and $z^{\prime}$ be two distinct elements of $\mathcal{S}^{1}$ and let $z_{1}, z_{2}, z_{1}^{\prime}$ and $z_{2}^{\prime}$ be the elements of $\mathcal{S}^{1}$ such that $f\left(z_{1}\right)=f\left(z_{2}\right)=z$ and $f\left(z_{1}^{\prime}\right)=f\left(z_{2}^{\prime}\right)=z^{\prime}$. Then $\left\{z_{1}\right\},\left\{z_{2}\right\}$ and $\left\{z_{1}, z_{2}\right\}$ belong to $\mathcal{F}_{n}(f)^{-1}(\{z\})$. Thus, $\mathcal{F}_{n}(f)$ is not a simple map. Also, $q_{\mathcal{S}^{1}}^{n}\left(\left\{z_{1}, z_{1}^{\prime}\right\}\right), q_{\mathcal{S}^{1}}^{n}\left(\left\{z_{1}, z_{2}^{\prime}\right\}\right), q_{\mathcal{S}^{1}}^{n}\left(\left\{z_{2}, z_{1}^{\prime}\right\}\right)$ and $q_{\mathcal{S}^{1}}^{n}\left(\left\{z_{2}, z_{2}^{\prime}\right\}\right)$ all belong to $\mathcal{S} \mathcal{F}_{n}(f)^{-1}\left(q_{\mathcal{S}^{1}}^{n}\left(\left\{z, z^{\prime}\right\}\right)\right)$. Hence, $\mathcal{S} \mathcal{F}_{n}(f)$ is not a simple map.

TheOREM 11.3. Let $X$ and $Y$ be continua, let $n$ be an integer greater than or equal to 2 , and let $f: X \rightarrow Y$ be a surjective map. If $\mathcal{S F}_{n}(f)$ is a simple map, then either $f$ is a homeomorphism or $f$ identifies two points. In particular, $f$ is a simple map.

Proof. Assume $\mathcal{S F}_{n}(f)$ is a simple map. If $\mathcal{S F}_{n}(f)^{-1}\left(F_{Y}^{n}\right)=\left\{F_{X}^{n}\right\}$, then $f$ is a homeomorphism by Lemma 3.7. Suppose there exists $\chi \in$ $\mathcal{S F}_{n}(f)^{-1}\left(F_{Y}^{n}\right) \backslash\left\{F_{X}^{n}\right\}$. Then $\left(q_{X}^{n}\right)^{-1}(\chi) \in \mathcal{F}_{2}(X) \backslash \mathcal{F}_{1}(X)$. Otherwise, we could find $k$ points $x_{1}, \ldots, x_{k}$ in $X(k \geq 3)$ such that $q_{X}^{n}\left(\left\{x_{1}, \ldots, x_{k}\right\}\right)=\chi$ and we would have that $q_{X}^{n}\left(\left\{x_{1}, x_{2}\right\}\right), q_{X}^{n}\left(\left\{x_{1}, x_{3}\right\}\right)$ and $q_{X}^{n}\left(\left\{x_{2}, x_{3}\right\}\right)$ would all belong to $\mathcal{S F}_{n}(f)^{-1}\left(F_{Y}^{n}\right)$, a contradiction. Assume that $\left(q_{X}^{n}\right)^{-1}(\chi)=\left\{x_{1}, x_{2}\right\}$. Observe that the previous argument implies that $\left.f\right|_{X \backslash\left\{x_{1}, x_{2}\right\}}$ is one-to-one. Therefore, $f$ identifies $x_{1}$ and $x_{2}$.

The following example shows that there exists a simple map $f$ between continua such that $\mathcal{F}_{n}(f)$ is not a simple map and $\mathcal{S F}_{n}(f)$ is a simple map.

ExAMPLE 11.4. Let $f:[0,1] \rightarrow \mathcal{S}^{1}$ be given by $f(t)=(\sin (2 \pi t), \cos (2 \pi t))$. Note that $f$ identifies two points. Then by Theorem 11.1 , we have that $\mathcal{F}_{n}(f)$ is not simple. It is easy to see that $\mathcal{S F}_{n}(f)$ is simple.

Theorem 11.5. Let $X$ and $Y$ be continua, let $n$ be an integer greater than or equal to 2 , and let $f: X \rightarrow Y$ be a surjective map. If there exist two 
elements $y_{1}$ and $y_{2}$ of $Y$ such that both $\mathcal{F}_{n}(f)^{-1}\left(\left\{y_{1}\right\}\right)$ and $\mathcal{F}_{n}(f)^{-1}\left(\left\{y_{2}\right\}\right)$ have two elements, then $\mathcal{S F}_{n}(f)$ is not simple.

Proof. Let $y_{1}$ and $y_{2}$ be two elements of $Y$, let $A_{1}$ and $B_{1}$ be two distinct elements in $\mathcal{F}_{n}(f)^{-1}\left(\left\{y_{1}\right\}\right)$, and let $A_{2}$ and $B_{2}$ be two distinct elements in $\mathcal{F}_{n}(f)^{-1}\left(\left\{y_{2}\right\}\right)$. Without loss of generality, we assume that $A_{1} \not \subset B_{1}$ and $A_{2} \not \subset B_{2}$. Let $a_{1} \in A_{1} \backslash B_{1}, b_{1} \in B_{1}, a_{2} \in A_{2} \backslash B_{2}$ and $b_{2} \in B_{2}$. Then $F_{X}^{n}$, $q_{X}^{n}\left(\left\{a_{1}, b_{1}\right\}\right)$ and $q_{X}^{n}\left(\left\{a_{2}, b_{2}\right\}\right)$ belong to $\mathcal{S F}_{n}(f)^{-1}\left(F_{Y}^{n}\right)$. Therefore, $\mathcal{S F}_{n}(f)$ is not simple.

TheOREM 11.6. Let $X$ and $Y$ be continua, let $n$ be an integer greater than or equal to 2, and let $f: X \rightarrow Y$ be a surjective map. Consider the following statements:

(1) $f$ is simple;

(2) $\mathcal{F}_{n}(f)$ is simple;

(3) $\mathcal{S F}_{n}(f)$ is simple.

Then (2) implies (1) and (3), (3) implies (1), (3) does not imply (2); and (1) does not imply either (2) or (3).

Proof. If $\mathcal{F}_{n}(f)$ is simple, then $f$ is a homeomorphism by Theorem 11.1. Thus, $\mathcal{S F}_{n}(f)$ is a homeomorphism ([2, Theorem 3.3]). Hence, $f$ and $\mathcal{S F}_{n}(f)$ are both simple maps. It follows from Theorem 11.3 that if $\mathcal{S F}_{n}(f)$ is a simple map, then $f$ is a simple map. The fact that (3) does not imply (2), follows from Example 11.4, and the fact that (1) does not imply either (2) or (3), follows from Example 11.2.

\section{REFERENCES}

[1] F. Barragán, On the n-fold symmetric product suspensions of a continuum, Topology Appl. 157 (2010), 597-604.

[2] F. Barragán, Induced maps on n-fold symmetric product suspensions, Topology Appl. 158 (2011), 1192-1205

[3] F. Barragán, Aposyndetic properties of the n-fold symmetric product suspension of a continuum, Glas. Mat. Ser. III 49(69) (2014), 179-193.

[4] J. Camargo and S. Macías, On freely decomposable maps, Topology Appl. 159 (2012), 891-899.

[5] J. J. Charatonik, On feebly monotone and related classes of mappings, Topology Appl. 105 (2000), 15-29.

[6] J. Dugundji, Topology, Allyn and Bacon, Inc., Boston, 1966.

[7] G. R. Gordh, Jr. and C. B. Hughes, On freely decomposable mappings of continua, Glas. Mat. Ser. III 14(34) (1979), 137-146.

[8] H. Hosokawa, Induced mappings on hyperspaces, Tsukuba J. Math. 21 (1997), 239250.

[9] G. Higuera and A. Illanes, Induced mappings on symmetric products, Topology Proc. 37 (2010), 367-401.

[10] S. Macías, On symmetric products of continua, Topology Appl. 92 (1999), 173-182.

[11] S. Macías, Aposyndetic properties of symmetric products of continua, Topology Proc. 22 (1997), 281-296. 
[12] S. Macías, Induced maps on $n$-fold hyperspace suspensions, Topology Proc. 28 (2004), $143-152$.

[13] S. Macías, Topics on continua, Chapman \& Hall/CRC, Boca Raton, 2005.

[14] J. M. Martínez-Montejano, Mutual aposyndesis of symmetric products, Topology Proc. 24 (1999), 203-213.

[15] S. B. Nadler, Jr., Hyperspaces of sets, Marcel Dekker, New York, Basel, 1978. Reprinted in: Aportaciones Matemáticas de la Sociedad Matemática Mexicana, Serie Textos \# 33, 2006.

[16] S. B. Nadler, Jr., A fixed point theorem for hyperspace suspensions, Houston J. Math. 5 (1979), 125-132.

F. Barragán

Instituto de Física y Matemáticas, Universidad Tecnológica de la Mixteca Carretera a Acatlima, Km. 2.5, Huajuapan de León, Oaxaca, C. P. 69000 México

E-mail: franco@mixteco.utm.mx

S. Macías

Instituto de Matemáticas, Universidad Nacional Autónoma de México

Circuito Exterior, Ciudad Universitaria, México D. F., C. P. 04510

México

E-mail: sergiom@matem.unam.mx

J. F. Tenorio

Current address:

Instituto de Matemáticas, Universidad Nacional Autónoma de México

Circuito Exterior, Ciudad Universitaria, México D. F., C. P. 04510

México

Permanent address:

Instituto de Física y Matemáticas, Universidad Tecnológica de la Mixteca Carretera a Acatlima, Km. 2.5, Huajuapan de León, Oaxaca, C. P. 69000 México

E-mail: jtenorio@mixteco.utm.mx

Received: 14.8.2014. 